\title{
Modeling heat transfer during friction stir welding using a meshless particle method
}

\author{
Yihua Xiao, ${ }^{\mathrm{a}, \mathrm{b}}$, Haifei Zhan ${ }^{\mathrm{b}}$, Yuantong $\mathrm{Gu}^{\mathrm{b}}$, Qinghua $\mathrm{Li}^{\mathrm{c}}$ \\ a School of Mechatronics Engineering, East China Jiaotong University, Nanchang 330013, China \\ ${ }^{\mathrm{b}}$ School of Chemistry, Physics and Mechanical Engineering, Queensland University of Technology, GPO Box \\ 2434, Brisbane, QLD 4001, Australia \\ c School of Civil Engineering and Architecture, East China Jiaotong University, Nanchang 330013, China
}

\begin{abstract}
Modeling heat transfer during friction stir welding (FSW) process is crucial for understanding welding mechanism and optimizing process parameters. Since heat transfer is usually accompanied with the material flow in FSW, the meshless method, which can easily treat large deformation in a Lagrangian framework, is promising for FSW modeling. In this paper, we develop a meshless particle method for the analysis of transient heat transfer during FSW process. In the developed method, a heat source model based on sticking friction is implemented to describe the heat generation of FSW. A particle approximation with first-order consistency is employed to discretize the governing equation of heat transfer. A penalty method is proposed to impose different thermal boundary conditions, and a smoothing algorithm is introduced to enhance numerical stability. Two examples are firstly given to verify the accuracy and parametric effect of the meshless particle method. The method is then used to simulate heat transfer during FSW of 12.7mm-thick Al6061-T6 plates. The calculated temperature distributions are presented and compared with those computed by FEM. The obtained thermal cycles are found to be in good agreement with those obtained from experiments. The validated model of FSW of Al6061-T6 plates is then employed to predict the maximum temperature, heat generation rate and torque for various welding parameters and tool dimensions.
\end{abstract}

Keywords: Heat transfer; Friction stir welding; Meshless particle method

\section{Introduction}

Friction stir welding (FSW) [1] is a renowned solid-state joining technology. Understanding of heat transfer during the FSW process is of great importance to FSW researchers because it largely determines the weld performance. Experimental measurement has been extensively used to study the thermal process of FSW. However, it suffers from several limitations, such as difficulties in testing temperature of stir zone and limited data of the temperature field. Alternatively, numerical modeling is an effective avenue in understanding such thermal process. In this regard, many numerical simulations [2-13] have been carried out to explore the temperature distribution and other physics that is involved in FSW. Various approaches including finite element method (FEM) [2-4], finite difference method [5-7] and meshless methods [8-13] have been utilized in these numerical simulations. Owing to the flexibility in space discretization and the capability of 


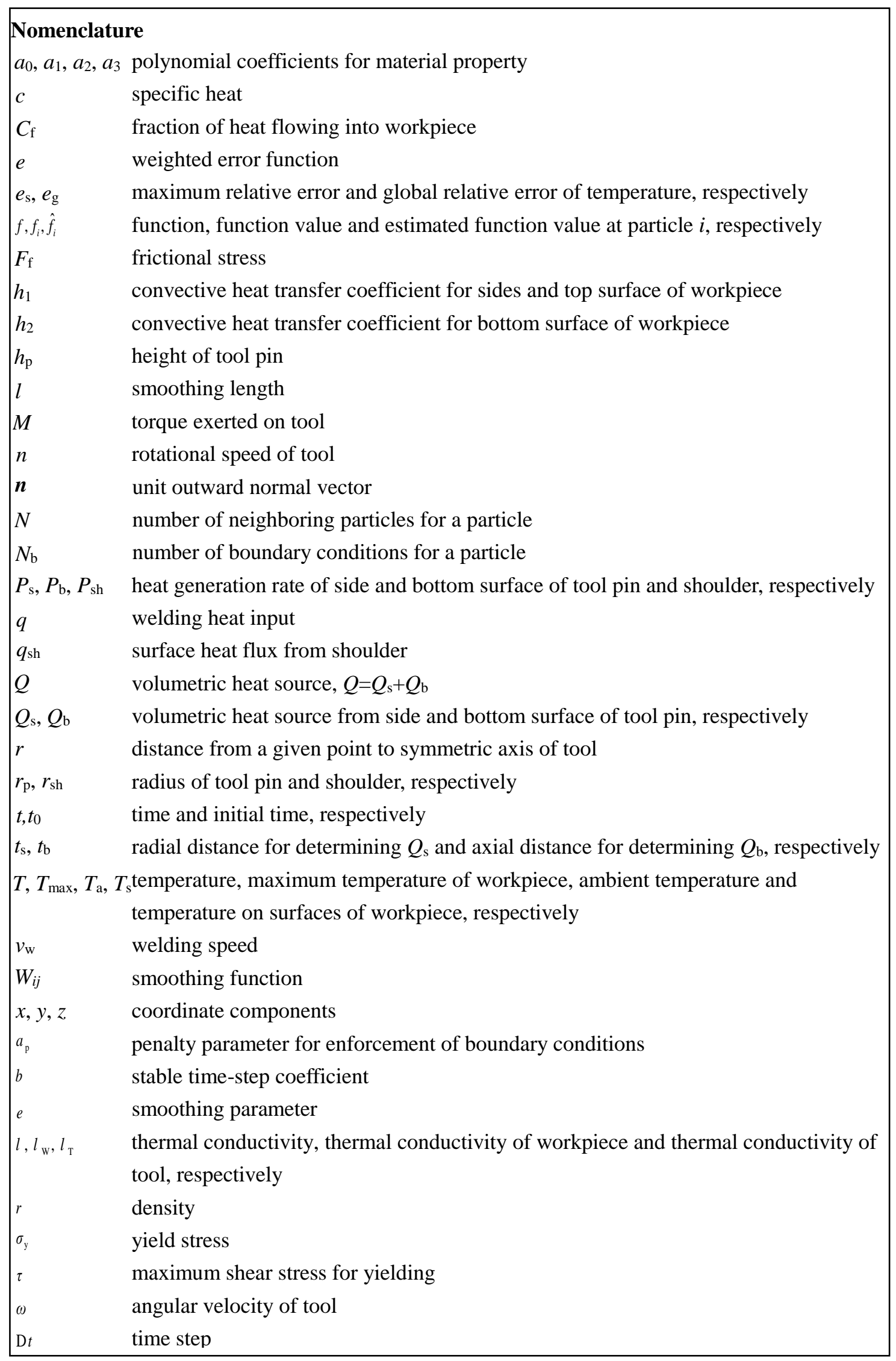

modeling large deformations in a Lagrangian framework, the meshless method appears as a promising tool in FSW modeling, especially in the coupled thermal-mechanical analysis of FSW 
for predicting temperature distribution and material flow simultaneously.

Meshless methods use points without connectivity for calculation. They provide advantages over FEM in many aspects, e.g., could easily treat complex geometries and incorporate adaptivity, could handle large deformation and discontinuity, and have good accuracy and high convergence rate. Currently, there are a number of meshless methods proposed, such as smoothed particle hydrodynamics (SPH) method [14], element-free Galerkin (EFG) method [15], reproducing kernel particle method (RKPM) [16], meshless local Petrov-Galerkin (MLPG) method [17] and point interpolation method [18]. These methods have been widely applied to fluid and solid mechanics problems, and are especially popular for problems involving large displacements and deformations like fluid flow [19], impact [20] and explosion [21]. In heat transfer field, meshless methods have also been extensively investigated and used. A. Singh , I.V. Singh and Prakash [22-25] have done important fundamental works on EFG method for heat transfer application. Singh, Tanaka and Endo [26-32] made a number of contributions to thermal analysis of composites especially carbon nanotube (CNT) composites based on EFG method. Wu and Tao [33] applied MLPG method to 2D steady-state heat transfer problems with irregular complex domain. Thakur et al. [34, 35] employed MLPG method to solve nonlinear heat transfer in irregular domains and phase change problems. Tian and Rao [36] proposed a MLPG method for solving steady-state nonlinear heat transfer problems with boundary conditions including heat flux, convection and radiation.

In recent years, a few meshless methods including natural element method (NEM) [8], adaptive EFG method [9] and SPH method [10-13] have been extended to model FSW process. Among these meshless methods, SPH method is the most popular one. This mainly benefits from its attractive features. Firstly, SPH method is a truly meshless method, which avoids numerical integration and the use of background meshes. Secondly, particle approximation for function and its derivatives in SPH method can be simply achieved by a summation over particles. Moreover, SPH method is easy to be implemented for high dimensional problems and to incorporate models for complex physics. However, SPH method also has its own deficiencies. One is the relatively low accuracy for particle approximation, especially for boundary particles and non-uniform particles. To overcome this problem, different modified methods have been proposed, such as RKPM [16], corrected smoothed particle method (CSPM) [37], finite particle method (FPM) [38], modified smoothed particle hydrodynamics (MSPH) [39] and symmetric smoothed particle hydrodynamics (SSPH) [40]. Among these modifications, SSPH shows attractive characteristics, which include the symmetry of matrix, no requirement of differentiating kernel function and the approximation of function and its derivatives obtained simultaneously. Another deficiency of SPH method is the inconvenience in dealing with boundary conditions. Very few studies have highlighted the enforcement of boundary conditions for SPH modeling of heat transfer. Monaghan et al. [41] discussed the treatment of temperature-specified boundaries and adiabatic boundaries (no need of treatment). Jeong et al. [42] presented a method, which needs the use of ghost particles, to implement temperature-specified and heat flux-specified boundary conditions. 
In this paper, we develop a meshless particle method to simulate the nonlinear transient heat transfer process of FSW. The method has the advantages of being truly meshless and easy implementation like SPH. Meanwhile, it has an improved particle approximation and an adequate method to treat various thermal boundary conditions conveniently. Specifically, the present method uses a set of particles without any connectivity to represent a problem domain, and directly enforces governing equations at each particle. It employs a particle approximation having first-order consistency to accurately approximate the field function and its derivatives. A penalty method, which avoids the use of ghost particles, is proposed to deal with all kinds of thermal boundary conditions involved in FSW process. A smoothing algorithm based on temperature change rate is introduced to ensure numerical stability. A heat source model based on sticking friction is derived and implemented to describe the heat generation in FSW process. The developed meshless particle method is finally applied to the simulation of temperature distributions during FSW of Al6061-T6 plates. It is worth mentioning that similar meshless framework has also been used in our previous work [43], which has focused on the modeling of tungsten inert gas (TIG) welding of stainless steel, while this work proposes its first-time application to the modelling of FSW. Also, in comparison with our previous work [43], the current method has several new features and improvements, such as new treatment of boundary condition, modified smoothing algorithm and simplified particle approximation. Moreover, a more complex heat source model for FSW modeling is incorporated.

The rest of the paper is organized as follows. Section 2 describes the mathematical model for heat transfer during FSW process. Section 3 details the formulation of the meshless particle method. Section 4 presents two test cases to verify the accuracy and parametric effect of the method. Section 5 gives the numerical model and results for FSW of Al6061-T6 plates. The last section draws the main conclusions of this work.

\section{Mathematical model for heat transfer during FSW process}

\subsection{Governing equation}

Fig. 1 shows the schematic diagram for heat transfer model of FSW butt welding of two workpieces. The governing equation for the heat transfer process is given as

$$
r c \frac{\partial T}{\partial t}=\frac{\partial}{\partial x}\left(l \frac{\partial T}{\partial x}\right)+\frac{\partial}{\partial y}\left(l \frac{\partial T}{\partial y}\right)+\frac{\partial}{\partial z}\left(l \frac{\partial T}{\partial z}\right)+Q
$$

where $r$ is the density, $c$ is the specific heat, $T$ is the temperature, $t$ is the time, $l$ is the thermal conductivity, $x, y$ and $z$ are the coordinates, respectively, and $Q$ is the volumetric heat source term consisting of two parts, i.e., $Q_{\mathrm{s}}$ and $Q_{\mathrm{b}}$, which represent the heat resulted from the friction between the tool pin's side and the workpiece and the friction between the tool pin's bottom surface and the workpiece, respectively. 


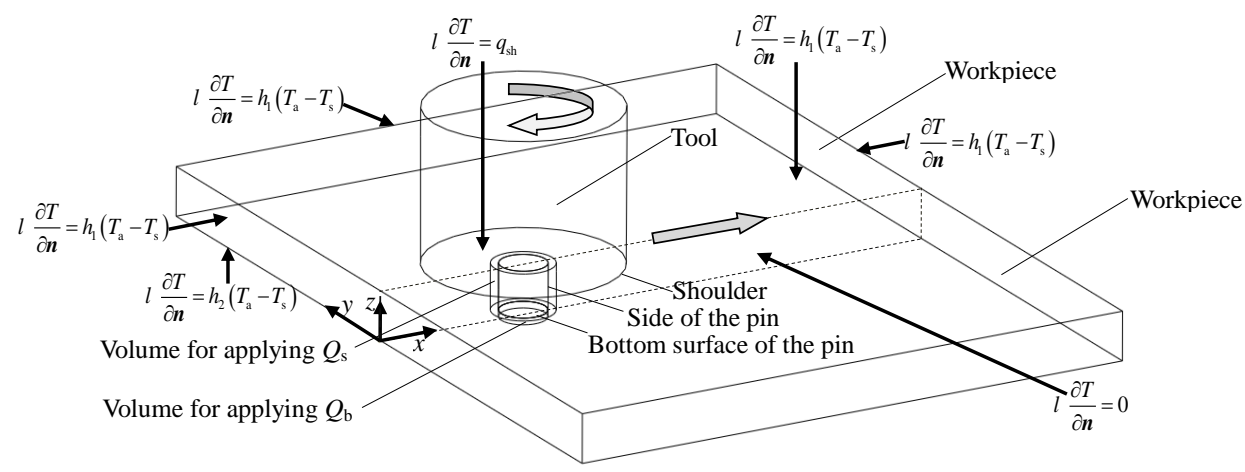

Fig. 1 Schematic diagram for heat transfer model of FSW process

\subsection{Boundary conditions}

Considering the symmetry of the problem, only one of the two workpieces is modeled to save computational cost. The boundary conditions for different surfaces of the workpiece are shown in Fig. 1.

The workpiece's sides (except for the one in the middle) and its top surface out of the tool shoulder expose to the surroundings. For these surfaces, convective boundary conditions are prescribed

$$
l \frac{\partial T}{\partial \boldsymbol{n}}=h_{1}\left(T_{\mathrm{a}}-T_{\mathrm{s}}\right)
$$

where $\boldsymbol{n}$ is the unit outward normal vector, $h_{1}$ is the convective heat transfer coefficient, $T_{\mathrm{a}}$ is the ambient temperature, and $T_{\mathrm{s}}$ is the temperature on the surfaces. The bottom surface of the workpiece is also treated as a convective boundary for simplicity $[3,5,6]$. The form of its boundary condition is the same as Eq. (2). An effective convective heat transfer coefficient, $h_{2}$, is specified for the bottom surface to consider the heat lost through its contact with backing plate.

The top surface of the workpiece within the tool shoulder is treated as boundary with specified surface heat flux. The boundary condition can be written as

$$
l \frac{\partial T}{\partial \boldsymbol{n}}=q_{\mathrm{sh}}
$$

where $q_{\text {sh }}$ is the surface heat flux representing the heat generated by the friction between the tool shoulder and the workpiece.

The workpiece's side in the middle is regarded as an adiabatic boundary, whose boundary condition is given as

$$
l \frac{\partial T}{\partial n}=0
$$

\subsection{Heat source model}

The heat generation during FSW process is characterized by the volumetric heat source terms $Q_{\mathrm{s}}$ and $Q_{\mathrm{b}}$ and the surface heat flux $q_{\mathrm{sh}}$. To derive their expressions, the following assumptions are adopted: (a) Heat is generated by the friction on the interfaces of the tool and the 
workpiece; (b) The tool and the workpiece are fully sticking at the interfaces, and the frictional stress on the interfaces, $F_{\mathrm{f}}$, equals the maximum shear stress for yielding, $\tau(T)$, namely

$$
F_{\mathrm{f}}=\tau(T)=\sigma_{\mathrm{y}}(T) / \sqrt{3}
$$

where $\sigma_{\mathrm{y}}$ is the yield stress; (c) The frictional work is completely converted to heat which enters into both the tool and the workpiece, and the fraction of the heat flowing into the workpiece is a constant $C_{\mathrm{f}}$. Note that the assumption of frictional stress equal to the maximum shear stress for yielding is similar as that used in the thermal pseudomechanical model developed by Schmidt and Hattel [44], which assume that the contact stress on the interface between the tool and the workpiece must balance with the maximum shear stress for yielding during the steady state of FSW. Such an assumption leads to an automatically stabilized effect on the temperature variation and a maximum temperature under the melting point (as is seen in Section 5). Also, it reduces the number of parameters required in the thermal modeling of FSW.

Fig. 2 shows a vertical view of the side of the tool pin. For an arbitrary point $A$ on the side, an infinitesimal cylindrical surface $d s$ adjacent to the point is considered. The infinitesimal surface $d s$ is represented by the arc $A A^{\prime}$ in the figure. It has a central angle of $d \theta$ for its base and a height of $d z$ in the axial direction of the tool. The heat generation rate on $d s$ can be expressed as

$$
d P_{\mathrm{s}}=\left(F_{\mathrm{f}} d s\right)\left(\omega r_{\mathrm{p}}\right)=\tau(T) \omega r_{\mathrm{p}}^{2} d \theta d z
$$

where $\omega$ is the angular velocity, and $r_{\mathrm{p}}$ is the radius of the tool pin. The heat generation rate imposed on the workpiece is obtained as $C_{\mathrm{f}} d P_{\mathrm{s}}$. It can not be applied as a surface heat flux to the workpiece, because the mechanical interactions between the workpiece and the pin are not simulated in the current thermal analysis, and the workpiece surface interacting with the pin is not available. Thus, a volumetric heat source $Q_{\mathrm{s}}$ is used to account for the effect of the heat generated by the tool pin's side. Assuming the heat for the workpiece $\left(C_{\mathrm{f}} d P_{\mathrm{s}}\right)$ at point $A$ dissipates uniformly in its neighboring volume $d V_{\mathrm{s}}$, which is an extrusion of $d s$ along the radial direction for a distance of $t_{\mathrm{s}}$, yields the volumetric heat source $Q_{\mathrm{s}}$, which can be calculated as

$$
Q_{\mathrm{s}}=\frac{C_{\mathrm{f}} d P_{\mathrm{s}}}{d V_{\mathrm{s}}}=\frac{2 C_{\mathrm{f}} \tau(T) \omega r_{\mathrm{p}}^{2}}{\dot{\mathrm{e}}^{\left.\dot{g}_{\mathrm{p}}+t_{\mathrm{s}}\right)^{2}-r_{\mathrm{p}}^{2} \stackrel{\mathrm{u}}{\mathrm{u}}}}
$$




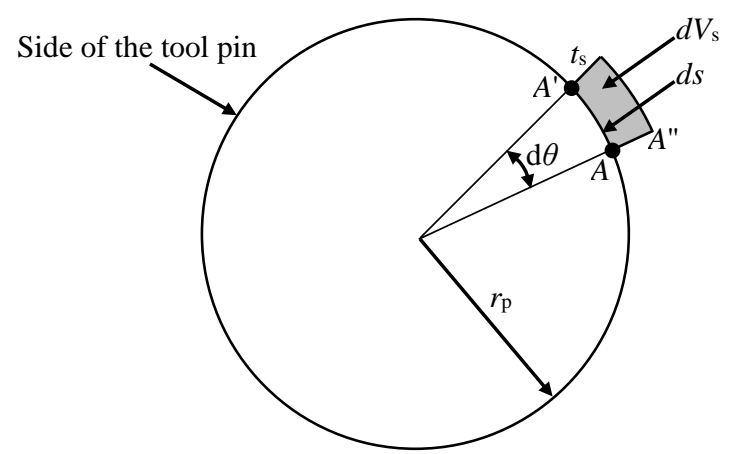

Fig. 2 Schematic diagram for heat generation by the side of the tool pin

In a similar way, the expression for $Q_{\mathrm{b}}$ can be derived. Fig. 3 is a vertical view of the bottom surface of the tool pin. For an arbitrary point $B$ on the surface, an infinitesimal area $d s$ (the dark shade in the Fig. 3) adjacent to the point is considered. The heat generation rate on the $d s$ can be expressed as

$$
d P_{\mathrm{b}}=\left(F_{\mathrm{f}} d s\right)(\omega r)=\tau(T) \omega r^{2} d r d \theta \quad\left(r £ r_{\mathrm{P}}\right)
$$

where $r$ is the distance from the point to the symmetric axis of the tool. The heat generation rate exerted on the workpiece is $C_{\mathrm{f}} d P_{\mathrm{b}}$. Assuming the heat dissipates uniformly in the neighboring volume $d V_{\mathrm{b}}$, which is a downward extrusion of the $d s$ along the axial direction for a distance of $t_{\mathrm{b}}$, gives the volumetric heat source $Q_{\mathrm{b}}$, which can be expressed as

$$
Q_{\mathrm{b}}=\frac{C_{\mathrm{f}} d P_{\mathrm{b}}}{d V_{\mathrm{b}}}=\frac{C_{\mathrm{f}} \tau(T) \omega r}{t_{\mathrm{b}}} \quad\left(r £ r_{\mathrm{P}}\right)
$$

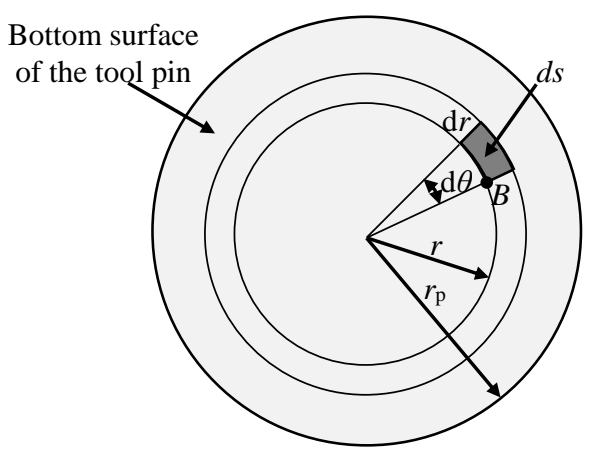

Fig.3 Schematic diagram for heat generation by the bottom surface of the pin

For an arbitrary point on the tool shoulder, an infinitesimal area $d s$ the same as that shown in Fig. 3 is taken for analyzing. The heat generation rate on the $d s$, which is denoted as $d P_{\text {sh }}$, has the same form of Eq. (8). The heat generation rate applied to the workpiece is $C_{\mathrm{f}} d P_{\mathrm{sh}}$. Then, the surface heat flux $q_{\mathrm{sh}}$ at the calculated point $B$ can be expressed as

$$
q_{\mathrm{sh}}=\frac{C_{\mathrm{f}} d P_{\mathrm{sh}}}{d s} \quad\left(r_{\mathrm{p}} £ r £ r_{\mathrm{sh}}\right)
$$


Substituting the right-hand side of Eq. (8) for $d P_{\mathrm{sh}}$ and the detailed expression for $d s$ in Eq. (10) leads to the final expression of $q_{\mathrm{sh}}$

$$
q_{\mathrm{sh}}=C_{\mathrm{f}} \tau(T) \omega r \quad\left(r_{\mathrm{p}} £ r £ r_{\mathrm{sh}}\right)
$$

\section{The meshless particle method}

\subsection{Particle approximation of first-order consistency}

To solve the governing equation, the problem domain is scattered with a set of particles, which have physical quantities, such as density, mass and temperature. A particle approximation having first-order consistency is constructed based on the particles, and employed to discretize the governing equation. In the particle approximation, the values of a function $f(\boldsymbol{x})$ at the neighboring particles of an arbitrary particle $i$ are estimated with the first-order Taylor series as

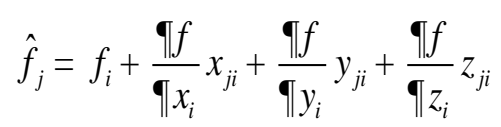

where $x_{j i}=x_{j}-x_{i}, y_{j i}=y_{j}-y_{i}, z_{j i}=z_{j}-z_{i}, \hat{f}_{j}$ is the estimated value of the function

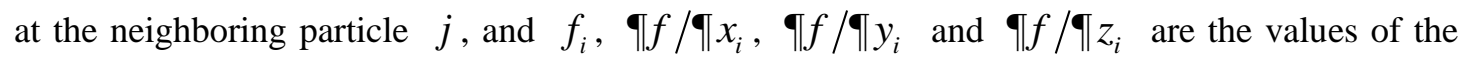
function and its derivatives at the calculated particle $i$. A weighted error function is defined as

$$
e=\stackrel{̊}{\mathrm{a}}_{j=1}^{N} W_{i j}\left(\hat{f}_{j}-f_{j}\right)^{2}
$$

where $f_{j}$ is the value of the function at particle $j, W_{i j}$ is the compactly supported smoothing function, and $N$ is the number of neighboring particles which are located in the effective domain of the smoothing function. To minimize the error function, setting its derivatives with respect to $f_{i}, \boldsymbol{\Psi} f / \boldsymbol{\Phi} x_{i}, \boldsymbol{q} f / \boldsymbol{\Phi} y_{i}$ and $\boldsymbol{\Psi} f / \boldsymbol{\Phi} z_{i}$ to zeros yields

$$
\left\{\begin{array}{l}
\sum_{j=1}^{N} W_{i j}\left(f_{i}+\frac{\partial f}{\partial x_{i}} x_{j i}+\frac{\partial f}{\partial y_{i}} y_{j i}+\frac{\partial f}{\partial z_{i}} z_{j i}\right)-\sum_{j=1}^{N} W_{i j} f_{j}=0 \\
\sum_{j=1}^{N} W_{i j}\left(f_{i}+\frac{\partial f}{\partial x_{i}} x_{j i}+\frac{\partial f}{\partial y_{i}} y_{j i}+\frac{\partial f}{\partial z_{i}} z_{j i}\right) x_{j i}-\sum_{j=1}^{N} W_{i j} f_{j} x_{j i}=0 \\
\sum_{j=1}^{N} W_{i j}\left(f_{i}+\frac{\partial f}{\partial x_{i}} x_{j i}+\frac{\partial f}{\partial y_{i}} y_{j i}+\frac{\partial f}{\partial z_{i}} z_{j i}\right) y_{j i}-\sum_{j=1}^{N} W_{i j} f_{j} y_{j i}=0 \\
\sum_{j=1}^{N} W_{i j}\left(f_{i}+\frac{\partial f}{\partial x_{i}} x_{j i}+\frac{\partial f}{\partial y_{i}} y_{j i}+\frac{\partial f}{\partial z_{i}} z_{j i}\right) z_{j i}-\sum_{j=1}^{N} W_{i j} f_{j} z_{j i}=0
\end{array}\right.
$$

Solving the above system of linear equations, we obtain the particle approximation of $f_{i}$ as

$$
f_{i}=\sum_{j=1}^{N} f_{j} \tilde{W}_{i j}
$$

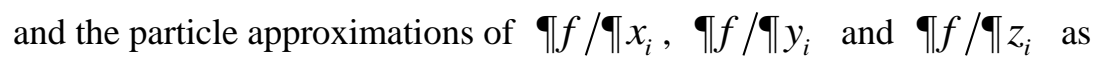




$$
\frac{\partial f}{\partial x_{i}}=\sum_{j=1}^{N} f_{j} \frac{\partial \tilde{W}_{i j}}{\partial x_{i}}, \frac{\partial f}{\partial y_{i}}=\sum_{j=1}^{N} f_{j} \frac{\partial \tilde{W}_{i j}}{\partial y}, \frac{\partial f}{\partial z_{i}}=\sum_{j=1}^{N} f_{j} \frac{\partial \tilde{W}_{i j}}{\partial z}
$$

where

$$
\left(\begin{array}{c}
\tilde{W}_{i j} \\
\frac{\partial \tilde{W}_{i j}}{\partial x} \\
\frac{\partial \tilde{W}_{i j}}{\partial y} \\
\frac{\partial \tilde{W}_{i j}}{\partial z}
\end{array}\right)=W_{i j}\left(\begin{array}{cccc}
\sum_{j=1}^{N} W_{i j} & \sum_{j=1}^{N} W_{i j} x_{j i} & \sum_{j=1}^{N} W_{i j} y_{j i} & \sum_{j=1}^{N} W_{i j} z_{j i} \\
\sum_{j=1}^{N} W_{i j} x_{j i} & \sum_{j=1}^{N} W_{i j} x_{j i}^{2} & \sum_{j=1}^{N} W_{i j} x_{j i} y_{j i} & \sum_{j=1}^{N} W_{i j} x_{j i} z_{j i} \\
\sum_{j=1}^{N} W_{i j} y_{j i} & \sum_{j=1}^{N} W_{i j} x_{j i} y_{j i} & \sum_{j=1}^{N} W_{i j} y_{j i}^{2} & \sum_{j=1}^{N} W_{i j} y_{j i} z_{j i} \\
\sum_{j=1}^{N} W_{i j} z_{j i} & \sum_{j=1}^{N} W_{i j} x_{j i} z_{j i} & \sum_{j=1}^{N} W_{i j} y_{j i} z_{j i} & \sum_{j=1}^{N} W_{i j} z_{j i}^{2}
\end{array}\right)^{-1}\left(\begin{array}{c}
1 \\
x_{j i} \\
y_{j i} \\
z_{j i}
\end{array}\right)
$$

Since $\sum_{j=1}^{N} \frac{\partial \tilde{W}_{i j}}{\partial x_{i}}=\sum_{j=1}^{N} \frac{\partial \tilde{W}_{i j}}{\partial y_{i}}=\sum_{j=1}^{N} \frac{\partial \tilde{W}_{i j}}{\partial z_{i}}=0$, the particle approximations of $\mathbb{\|} f / \boldsymbol{q} x_{i}, \boldsymbol{q} f / \boldsymbol{q} y_{i}$ and $\mathbb{q} f / \boldsymbol{\Phi} z_{i}$ can be rewritten as

$$
\frac{\partial f}{\partial x_{i}}=\sum_{j=1}^{N} f_{j i} \frac{\partial \tilde{W}_{i j}}{\partial x_{i}}, \frac{\partial f}{\partial y_{i}}=\sum_{j=1}^{N} f_{j i} \frac{\partial \tilde{W}_{i j}}{\partial y}, \frac{\partial f}{\partial z_{i}}=\sum_{j=1}^{N} f_{j i} \frac{\partial \tilde{W}_{i j}}{\partial z}
$$

where $f_{j i}=f_{j i}-f_{i}$.

In this work, the smoothing function $W_{i j}$ is chosen as the cubic spline function

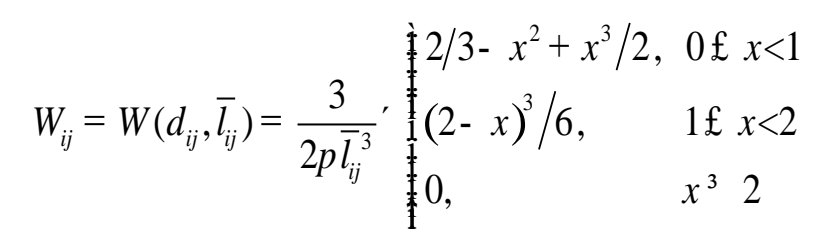

where $x=d_{i j} / \bar{l}_{i j}, d_{i j}$ is the distance between particle $i$ and $j, \bar{l}_{i j}=\left(l_{i}+l_{j}\right) / 2, l_{i}$ and $l_{j}$ are the smoothing length of particle $i$ and $j$, respectively.

It should be mentioned that particle approximations of Eqs. (15) and (16) have the same form as SSPH [40] if the first-order Taylor expansion is taken for function approximation in SSPH. Hence, the above particle approximation has the advantages of SSPH. However, the approach to deriving Eq. (14), which determines the particle approximations of Eqs. (15) and (16), is totally different from that presented in Reference [40]. With this new approach, we can easily find a penalty method for treating boundary conditions, which will be detailed in the later description. It should also be noted that the particle approximation in this paper excludes the particle volume compared with that used in our previous work [43]. This simplifies the calculation while maintains the first-order consistency of approximation.

\subsection{Discretization of the governing equation}

To discretize the governing equation, following the way in the work by Jeong et al. [42], we 
decompose it into two first-order partial differential equations, which are

$$
r c \frac{\partial T}{\partial t}=\frac{\partial q^{x}}{\partial x}+\frac{\partial q^{y}}{\partial y}+\frac{\partial q^{z}}{\partial z}+Q
$$

and

$$
q^{x}=l \frac{\partial T}{\partial x}, q^{y}=l \frac{\partial T}{\partial y}, q^{z}=l \frac{\partial T}{\partial z}
$$

where $q^{x}, q^{y}$ and $q^{z}$ are the heat fluxes.

Eqs. (20) and (21) are enforced at each particle. The first-order derivatives involved in the equations are discretized with Eq. (18), and the source term $Q$ is approximated with Eq. (15). Then, the following discrete governing equation is obtained

$$
\frac{\partial T_{i}}{\partial t}=\frac{1}{r_{i} c_{i}}\left(\sum_{j=1}^{N} q_{j i}^{x} \frac{\partial \tilde{W}_{i j}}{\partial x}+\sum_{j=1}^{N} q_{j i}^{y} \frac{\partial \tilde{W}_{i j}}{\partial y}+\sum_{j=1}^{N} q_{j i}^{z} \frac{\partial \tilde{W}_{i j}}{\partial z}+\sum_{j=1}^{N} Q_{j} \tilde{W}_{i j}\right)
$$

where

$$
q_{i}^{x}=l \sum_{j=1}^{N} T_{j i} \frac{\partial \tilde{W}_{i j}}{\partial x}, q_{i}^{y}=l \sum_{j=1}^{N} T_{j i} \frac{\partial \tilde{W}_{i j}}{\partial y}, q_{i}^{z}=l \sum_{j=1}^{N} T_{j i} \frac{\partial \tilde{W}_{i j}}{\partial z}
$$

\subsection{Enforcement of boundary conditions}

A penalty method is proposed to impose boundary conditions. Boundary conditions of different types described in Section 2.2 are uniformly expressed as

$$
\frac{\partial T}{\partial \boldsymbol{n}}=\frac{\partial T}{\partial x} \cos q_{x}+\frac{\partial T}{\partial y} \cos q_{y}+\frac{\partial T}{\partial z} \cos q_{z}=k
$$

where

$$
k= \begin{cases}h\left(T_{\mathrm{a}}-T_{\mathrm{s}}\right) / l, & \text { convective boundaries } \\ q / l, & \text { boundaries of specified surface heat flux } \\ 0, & \text { symmetric boundaries }\end{cases}
$$

To calculate the temperature derivatives in Eq. (21) for boundary particles, the above boundary conditions are taken into account by adding a penalty term to the weighed error function. Then, the error function for temperature becomes

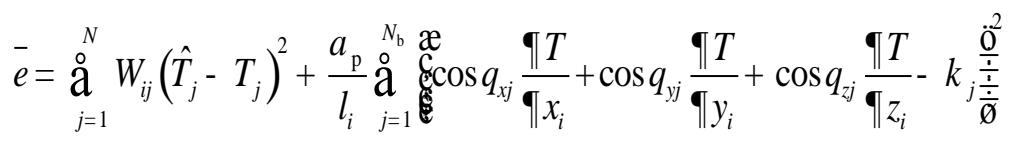

where $a_{\mathrm{p}}$ is the penalty parameter, $N_{\mathrm{b}}$ is the number of boundary conditions of particle $i$, and $\cos q_{x j}, \cos q_{y j}$ and $\cos q_{z j}$ are the direction cosines of the normal vector related to the $j$ th boundary condition of particle $i$. In a similar way stated in Section 3.1, minimizing the error function and solving the resulting system of linear equations give the particle approximation for the temperature derivatives of boundary particles 


$$
\left\{\begin{array}{l}
\frac{\partial T}{\partial x_{i}}=A_{21} F_{1}+A_{22} F_{2}+A_{23} F_{3}+A_{24} F_{4} \\
\frac{\partial T}{\partial y_{i}}=A_{31} F_{1}+A_{32} F_{2}+A_{33} F_{3}+A_{34} F_{4} \\
\frac{\partial T}{\partial z_{i}}=A_{41} F_{1}+A_{42} F_{2}+A_{43} F_{3}+A_{44} F_{4}
\end{array}\right.
$$

where

$$
\begin{aligned}
& \boldsymbol{A}=\stackrel{0}{\mathrm{a}}_{j}^{N} W_{i j} \boldsymbol{P}_{j} \boldsymbol{P}_{j}^{\mathrm{T}}+\frac{a_{\mathrm{p}}}{l_{i}} \stackrel{ }{\mathrm{b}}_{j}^{N_{\mathrm{b}}} \boldsymbol{Q}_{j} \boldsymbol{Q}_{j}{ }^{\mathrm{T}}
\end{aligned}
$$

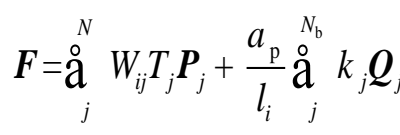

$$
\begin{aligned}
& \boldsymbol{P}_{j}=\operatorname{li}^{\prime} x_{j i} y_{j i} z_{j i} \mathrm{ul}_{\mathrm{G}}^{\mathrm{T}}
\end{aligned}
$$

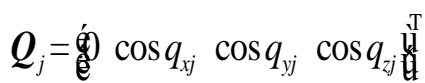

The penalty parameter $a_{\mathrm{p}}$ can be taken values in a wide range for general problems according our numerical tests. The larger $a_{\mathrm{p}}$ is, the more precise numerical result is. However, too large value may lead to a difficulty in numerical calculation. In this work, $a_{\mathrm{p}}$ is chosen as $10^{8}$ for all the calculations.

\subsection{Numerical stability}

There are moving heat sources and extensive changes of temperature during FSW process, which may cause numerical instability. To deal with this issue, a smoothing algorithm is introduced. The temperature change rate calculated by Eq. (22) is smoothed through the following particle approximation

$$
\frac{\partial T_{\mathrm{s} i}}{\partial t}=\sum_{j=1}^{N} \frac{\partial T_{j}}{\partial t} \tilde{W}_{i j}
$$

Then, a corrected temperature change rate is calculated as

$$
\frac{\partial T_{\mathrm{c} i}}{\partial t}=(1-e) \frac{\partial T_{i}}{\partial t}+e \frac{\partial T_{\mathrm{s} i}}{\partial t}
$$

where $e$ is a parameter used to adjust the smoothing extent, whose value is in the range of $[0,1]$. $\partial T_{\text {c } i} / \partial t$ is finally adopted to update temperature of particles. Note that Eq. (33) can be rewritten as

$$
\frac{\partial T_{\mathrm{c} i}}{\partial t}=\frac{\partial T_{i}}{\partial t}+e \sum_{j=1}^{N}\left(\frac{\partial T_{j}}{\partial t}-\frac{\partial T_{i}}{\partial t}\right) \tilde{W}_{i j}
$$

From this expression, it can be seen that the above correction is similar to the XSPH correction [45] for velocity which is commonly used in SPH. The above correction can enhance the stability 
of the meshless method and produce less influences on the accuracy compared with the smoothing for temperature used in our previous work [43].

\subsection{Time integration}

The Leap-Frog scheme [46] is employed to integrate the discrete governing equation (22) in time. At the end of the first time step, the temperature is advanced for half of a time step

$$
\left\{\begin{array}{l}
t=t_{0}+\mathrm{D} t \\
T_{i}\left(t_{0}+\frac{\mathrm{D} t}{2}\right)=T_{i}\left(t_{0}\right)+\frac{\mathrm{D} t}{2} \frac{\partial T_{\mathrm{c} i}\left(t_{0}\right)}{\partial t}
\end{array}\right.
$$

At the beginning of each subsequent time step, the temperature is predicted with

$$
T_{i}(t)=T_{i}\left(t-\frac{\mathrm{D} t}{2}\right)+\frac{\mathrm{D} t}{2} \frac{\partial T_{\mathrm{c} i}(t-\mathrm{D} t)}{\partial t},
$$

and at the end of each subsequent time step, the temperature is stepped forward as

$$
\left\{\begin{array}{l}
t=t+\mathrm{D} t \\
T_{i}\left(t+\frac{\mathrm{D} t}{2}\right)=T_{i}\left(t-\frac{\mathrm{D} t}{2}\right)+\mathrm{D} t \frac{\partial T_{\mathrm{c} i}(t)}{\partial t}
\end{array}\right.
$$

In this work, a fixed time step is used. The time step limit is estimated with the formula proposed by Cleary and Monaghan [41] as

$$
\mathrm{D} t=\beta c \rho l^{2} / \lambda
$$

where $\beta$ is a constant limited by the stability. The suitable value for $\beta$ will be discussed in Section 4.

\subsection{Solution procedure}

The following gives a general solution procedure of the present meshless particle method for transient heat transfer during FSW process:

(a) Generate particles for problem domain, initialize variables for each particle, prescribe boundary conditions for boundary particles, and set time step and total simulation time;

(b) Search neighboring particles for each particle;

(c) Calculate $\tilde{W}_{i j}, \partial \tilde{W}_{i j} / \partial x, \partial \tilde{W}_{i j} / \partial y$ and $\partial \tilde{W}_{i j} / \partial z$ with Eq. (17);

(d) Advance temperature for half of a time step with Eq. (35);

(e) Calculate $Q_{s}, Q_{b}$ and $q_{\text {sh }}$ with Eqs. (7), (9) and (11), respectively;

(f) Calculate heat fluxes $q^{x}, q^{y}$ and $q^{z}$ : For internal particles, use Eq. (23); For boundary particles, use Eq. (27) to obtain temperature derivatives, and then insert them into Eq. (21);

(g) Calculate volumetric heat source term $Q$ by $Q=Q_{\mathrm{s}}+Q_{\mathrm{b}}$;

(h) Calculate temperature change rate with Eq. (22);

(i) Calculate corrected temperature change rate with Eq. (33) or (34);

(j) Update temperature with Eq. (36) for the first time step and with Eq. (37) with subsequent 
time steps;

(k) If the time reaches the total simulation time, calculation is over; Otherwise, go to step 4.

The detailed calculation procedure for the volumetric heat source terms $Q_{\mathrm{s}}$ and $Q_{\mathrm{b}}$ and the surface heat flux $q_{\mathrm{sh}}$ is given as follows:

(a) Calculate the current location of the tool;

(b) Loop over particles:

- If a particle is in a hollow cylinder with the tool pin's side as the inner surface and a thickness of $t_{\mathrm{s}}$ (see the volume for applying $Q_{\mathrm{s}}$ in Fig. 1), the projection point of the particle on the tool pin's side along the radial direction is determined, and its temperature $T$ is approximated with its adjacent particles. Then, $\tau(T)$ at the projection point is obtained with the calculated temperature and the given material properties according to Eq. (5). Inserting the obtained $\tau(T)$ into Eq. (7) gives $Q_{\mathrm{s}}$ for the particle. If a particle is not in the hollow cylinder, $Q_{\mathrm{s}}$ for the particle is 0 .

- If a particle is in a cylinder with the tool pin's bottom surface as the top surface and a height of $t_{\mathrm{b}}$ (see the volume for applying $Q_{\mathrm{b}}$ in Fig. 1), the projection point of the particle on the tool pin's bottom surface along the axial direction is determined, and its temperature $T$ and distance to the symmetry axis of the tool $r$ are calculated. Then, $\tau(T)$ at the projection point is obtained with Eq. (5). Inserting the obtained $r$ and $\tau(T)$ into Eq. (9) gives $Q_{\mathrm{b}}$ for the particle. If a particle is not in the cylinder, $Q_{\mathrm{b}}$ for the particle is 0 .

- If a particle is on the top surface of the workpiece and within the shoulder, its distance to the symmetry axis of the tool $r$ are calculated. The temperature at the particle is used in Eq. (5) to determine the maximum shear stress for yielding $\tau(T)$. Inserting the obtained $r$ and $\tau(T)$ into Eq. (11) gives $q_{\mathrm{sh}}$ for the particle. If a particle is not on the top surface of the workpiece or within the shoulder, $q_{\mathrm{sh}}$ for the particle is 0 . If it is in the plunge phase of FSW, $q_{\mathrm{sh}}$ is set to 0 for all particles since the shoulder is not in contact with the top surface of the workpiece.

\section{Numerical validation}

This section presents numerical examples to validate the accuracy of the meshless particle method and study the effect of parameters in this method. SI units are assumed to be used throughout these numerical examples.

\subsection{Heat transfer in a solid cube with convective boundaries}

A solid cube with initial temperature $T_{0}=1$ is placed in the surrounding whose temperature 
maintains at 0 . The cube cools as the time elapses due to the convective heat transfer at its surfaces. The convective heat transfer coefficients at all surfaces are set to 1 . The centre of the cube is at the origin of the coordinate system. The side length of the cube is $2 a$, where $a$ is taken as 1 in the calculation. The material properties of the cube are $\rho=1, c=1$ and $\lambda=1$. The successive states of the cube during the whole cooling process can be analytically determined as [47]

$$
\begin{gathered}
T=f(x, t) f(y, t) f(z, t) \\
f(x, t)=\frac{\sin \left(n_{1} a\right)}{n_{1} a m_{1}} \cos \left(n_{1} x\right) \mathrm{e}^{-k\left(n_{1}\right)^{2} t}+\frac{\sin \left(n_{2} a\right)}{n_{2} a m_{2}} \cos \left(n_{2} x\right) \mathrm{e}^{-k\left(n_{2}\right)^{2} t}+\frac{\sin \left(n_{3} a\right)}{n_{3} a m_{3}} \cos \left(n_{3} x\right) \mathrm{e}^{-k\left(n_{3}\right)^{2} t}+\cdots
\end{gathered}
$$

where $k=l /(r c), n_{i}$ is calculated by the following equation

$$
n_{i} a \tan \left(n_{i} a\right)=\frac{h a}{l},
$$

and $m_{i}$ is

$$
m_{i}=\frac{1}{2}\left(1+\frac{\sin \left(2 n_{i} a\right)}{2 n_{i} a}\right) .
$$

The functions $f(y, t)$ and $f(z, t)$ are obtained in the same way.

To solve the problem with the present meshless particle method, $41 \times 41 \times 41$ uniform particles are used to discretize the cube. The corresponding particle spacing is 0.05 , and the smoothing length of each particle is 1.25 times of particle spacing. The effect of the smoothing parameter $e$ and the time step $\mathrm{D} t$ are studied by calculating the problem with various values of them. To evaluate the computational accuracy, maximum relative error and global relative error are defined as

$$
e_{\mathrm{s}}(t)=\max _{i}\left\{\left|\left(T_{\mathrm{p}}\left(\boldsymbol{x}_{i}, t\right)-T_{\mathrm{a}}\left(\boldsymbol{x}_{i}, t\right)\right) / T_{\mathrm{a}}\left(\boldsymbol{x}_{i}, t\right)\right|\right\}
$$

and

$$
e_{\mathrm{g}}(t)=\sqrt{\sum_{i=1}^{N P}\left[T_{\mathrm{p}}\left(\boldsymbol{x}_{i}, t\right)-T_{\mathrm{a}}\left(\boldsymbol{x}_{i}, t\right)\right]^{2} / \sum_{i=1}^{N P}\left[T_{\mathrm{a}}\left(\boldsymbol{x}_{i}, t\right)\right]^{2}},
$$

respectively, where $T_{\mathrm{p}}\left(\boldsymbol{x}_{i}, t\right)$ and $T_{\mathrm{a}}\left(\boldsymbol{x}_{i}, t\right)$ are the temperature of particle $i$ at the time $t$ from the meshless solution and the analytical solution, respectively, and $N P$ is the total number of particles.

Table 1 shows $e_{\mathrm{s}}$ and $e_{\mathrm{g}}$ at different time for various $e$ and $\mathrm{D} t$. It can be seen that all the results for different $e$ have small errors and exhibit good accuracy. In general, $e_{\mathrm{s}}$ decreases as $e$ increases, and $e_{\mathrm{g}}$ showes the same trend for $t=0.3$ and 0.4 (though it is slightly smaller for a small $e$ compared with a large one when $t=0.1$ and 0.2). Overall, using a larger value of $e$ tends to produce better results based on the current numerical test. Moreover, a larger $e$ leads to 
better stability for complex problems. Thus, we use 1 for $e$ in the following calculation. For $\mathrm{D} t$, we also tried different values. The results for two selected values are listed in Table 1 . When $\mathrm{D} t$ is taken as $2.0 \times 10^{-3}$ which is corresponding to about 0.5 for $\beta$, the calculation is still stable, and the computational accuracy is nearly the same as that for $\mathrm{D} t=1.0 \times 10^{-3}$. For other problems calculated in the following sections, it is also found that the stable time-step coefficient $\beta$ can be as large as up to about 0.5 . This suggests that a recommended value of $\beta$ for the present method could be 0.5 , which is relatively larger than 0.15 used in SPH [41].

Table 1 Effect of the time step $\mathrm{D} t$ and the smoothing parameter $e$

\begin{tabular}{|c|c|c|c|c|c|c|c|c|c|}
\hline $\mathrm{D} t$ & $e$ & $e_{\mathrm{s}}(0.1)$ & $e_{\mathrm{g}}(0.1)$ & $e_{\mathrm{s}}(0.2)$ & $e_{\mathrm{g}}(0.2)$ & $e_{\mathrm{s}}(0.3)$ & $e_{\mathrm{g}}(0.3)$ & $e_{\mathrm{s}}(0.4)$ & $e_{\mathrm{g}}(0.4)$ \\
\hline $2.0 \times 10^{-3}$ & 1.0 & 0.0053 & 0.0013 & 0.0054 & 0.0010 & 0.0051 & 0.0005 & 0.0052 & 0.0003 \\
\hline $2.0 \times 10^{-3}$ & 0.5 & 0.0067 & 0.0011 & 0.0063 & 0.0009 & 0.0058 & 0.0007 & 0.0057 & 0.0006 \\
\hline $2.0 \times 10^{-3}$ & 0.0 & 0.0073 & 0.0011 & 0.0064 & 0.0009 & 0.0059 & 0.0009 & 0.0057 & 0.0010 \\
\hline $1.0 \times 10^{-3}$ & 1.0 & 0.0053 & 0.0013 & 0.0054 & 0.0010 & 0.0051 & 0.0005 & 0.0052 & 0.0003 \\
\hline $1.0 \times 10^{-3}$ & 0.5 & 0.0067 & 0.0011 & 0.0062 & 0.0009 & 0.0058 & 0.0007 & 0.0057 & 0.0006 \\
\hline $1.0 \times 10^{-3}$ & 0.0 & 0.0073 & 0.0011 & 0.0064 & 0.0009 & 0.0059 & 0.0009 & 0.0057 & 0.0010 \\
\hline
\end{tabular}

Fig. 4 presents the calculated isothermals. The isothermals are for the top surface of the cube where the temperature change is most remarkable. It can be seen that the results of the present method are in excellent agreement with the analytical solutions.

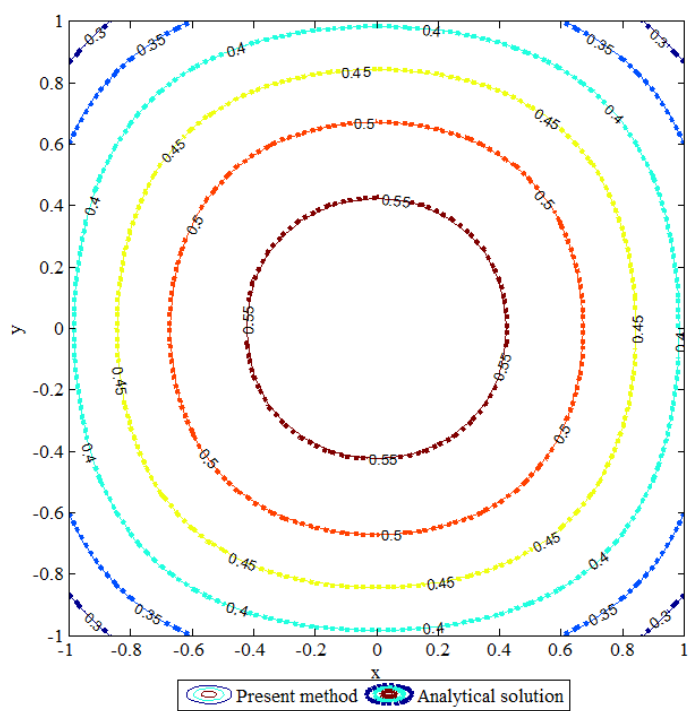

(a)

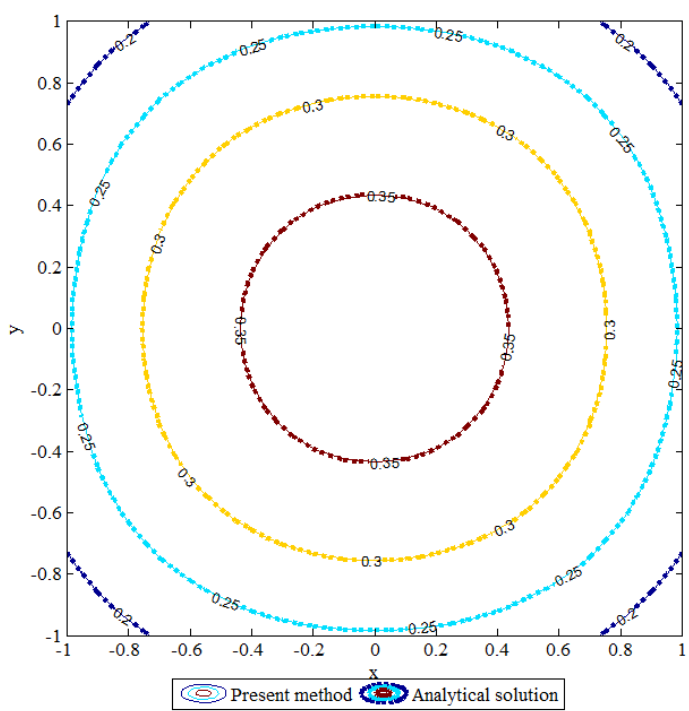

(b)

Fig. 4 Isothermals for the surface of $z=0$ : (a) $t=0.2$; (b) $t=0.4$ 
In order to show the effect of particle distribution on the results of the present meshless particle method, irregular particle discretization shown in Fig. 5(a) is also used to calculate the problem. The cube is discretized with 69419 irregular particles. The number of particles is close to that for the uniform particle discretization used in the previous calculation. The smoothing length of a particle is taken as 1.25 times of local particle spacing which is estimated as the average distance between the particle of interest and its three nearest neighboring particles. The estimated minimum and maximum local particle spacings are 0.027 and 0.095 , respectively. This implies that there is significant nonuniformity for the particle distribution. According the minimum local particle spacing, the time step is taken as $5.0 \times 10^{-4}$ to ensure the stability of calculation. Fig. 5 gives a comparison of the temperature distributions obtained with irregular and uniform particle distributions. It can be seen that the two temperature distributions are in good agreement with each other. Compared with the analytical solution, the result obtained with irregular particle distribution shows small relative errors of temperature, which are 0.0042 for $e_{\mathrm{s}}(0.4)$ and 0.0017 for $e_{\mathrm{g}}(0.4)$. The errors are close to those for uniform particle distribution listed in Table 1. The above discussion indicates that the present method works well for irregular particle discretization, and its accuracy is not sensitive to the particle distribution.

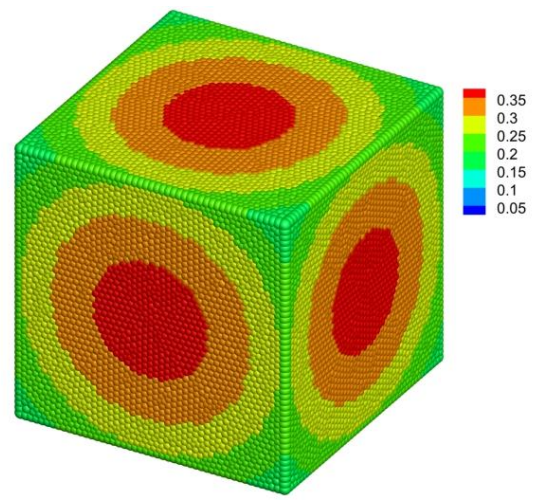

(a)

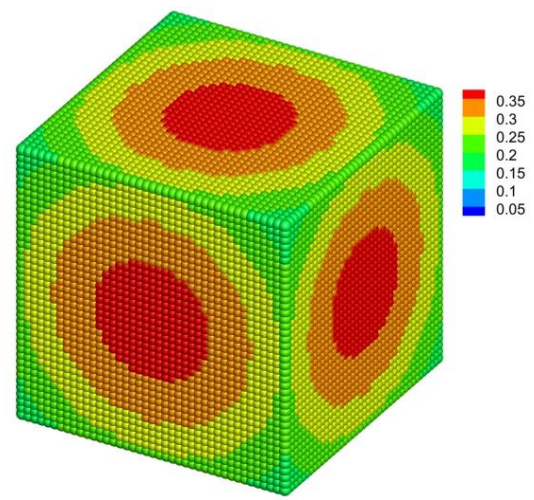

(b)

Fig. 5 Temperature distributions at $t=0.4$ for different particle distributions: (a) Irregular; (b) Uniform ( $\varepsilon=1.0$, $\left.\Delta t=2.0 \times 10^{-3}\right)$

\subsection{Heat transfer in a solid cube with mixed boundaries}

The cube with mixed boundary conditions imitating those in FSW is further employed to test the performance of the present method. As shown in Fig. 6, the left surface of the cube is an adiabatic boundary, and the other surfaces are convective boundaries. The convective heat transfer coefficient of the bottom surface is 10 , and those of the remained surfaces are 1 , which is much smaller than the former. Except for the aforementioned boundary conditions, all other calculation settings including initial temperature and material properties are the same as those in the previous example. The total simulation time is set to 0.4 . 


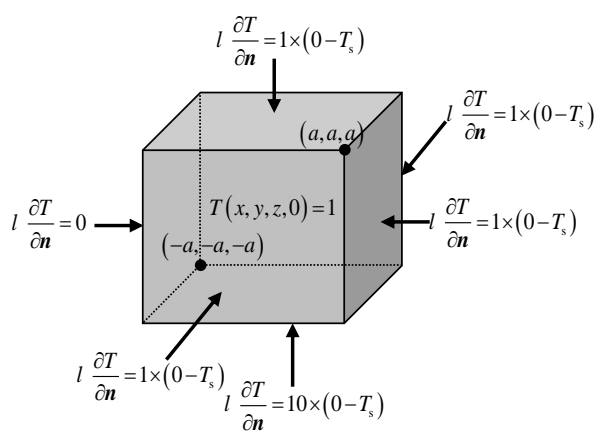

Fig. 6 Schematic diagram for heat transfer in a solid cube with mixed boundaries

Both the present method and FEM are used to calculate the problem. For the calculation of the present method, the cube is discretized with uniform particles with spacing of 0.05 , the smoothing length is taken as 1.25 times of particle spacing, and the time step is $2.0 \times 10^{-3}$. For the FEM calculation, ABAQUS/standard [48] is used as the solver. The cube is discretized with uniform hexahedron elements with side length of 0.05 , which is the same as the particle spacing for the meshless method. The element type is 8-node linear heat transfer brick element (DC3D8). The fixed time incrementation is used, and three time increments (i.e., time steps) including $1.0 \times 10^{-3}$, $1.0 \times 10^{-2}$ and $1.0 \times 10^{-1}$ are tested. All the three time increments are found to be able to produce stable results without convergence problems, but the result for the time increment of $1.0 \times 10^{-1}$ show large differences to those for the other two time increments, so it is assumed to be inaccurate and is not included in the following discussion.

Since it is generally believed that the FEM result is more accurate for smaller time increment, we use the FEM result with the smallest time increment $\left(1.0 \times 10^{-3}\right)$ as a reference solution to validate the result of the present method. Fig. 7 gives the temperature distributions calculated by the present method and FEM. It can be seen that the temperature distributions of the two methods agree well with each other. To quantatively compare the results of the two methods, Table 2 shows the relative errors of temperature. The relative errors between the meshless solution and the FEM solution with time increment of $1.0 \times 10^{-3}$ are very small. The above results demonstrate the feasibility of the present method for problems with complex boundary conditions. As for the computational cost, under the same computer configurations (Intel (R) Xeon (R) CPU E5-1650, 32GB RAM), the present method takes 20 minutes to complete the simulation, while the FEM uses about 49 minutes for the time increment of $1.0 \times 10^{-3}$. It should be mentioned that the FEM can utilize a much larger time increment to significantly reduce the computational time. For example, when a time increment of $1.0 \times 10^{-2}$ is used, the FEM calculation only takes about 5 minutes and becomes much faster than the meshless calculation. However, its accuracy also changes in some extent. This can be seen from the relative errors between the meshless solution and the FEM solution with time increment of $1.0 \times 10^{-2}$ as shown in Table 2. 


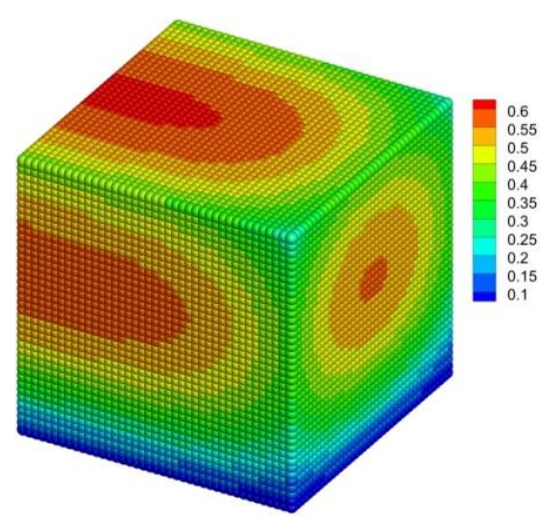

(a)

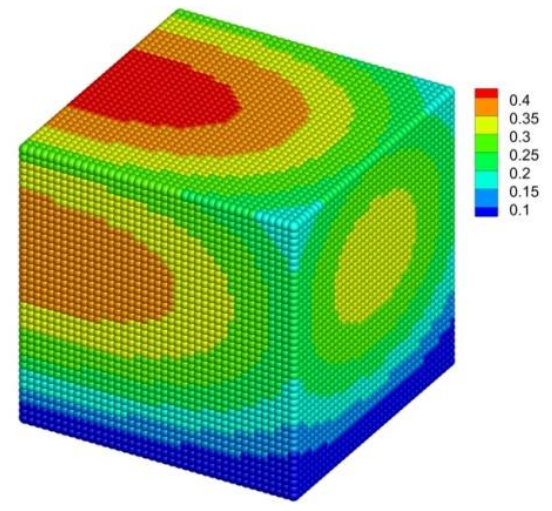

(c)

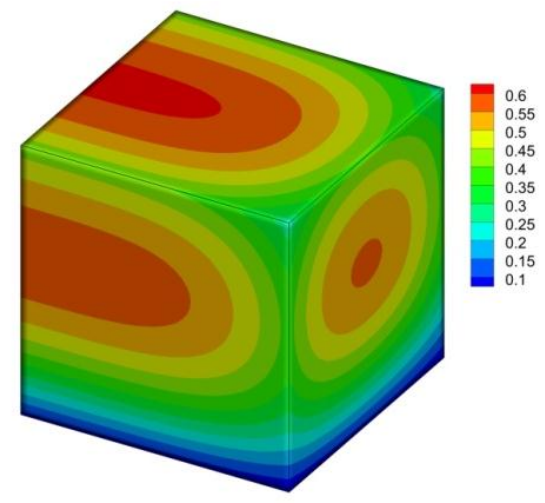

(b)

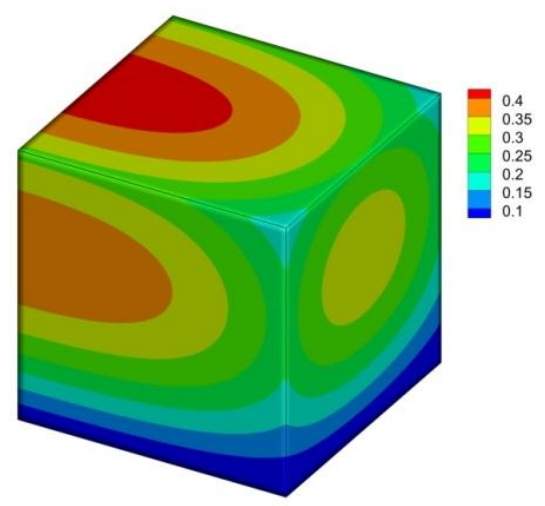

(d)

Fig. 7 Temperature distributions of the cube: (a) present method, $t=0.2$; (b) FEM with time increment of $1.0 \times 10^{-3}$, $t=0.2$; (c) present method, $t=0.4$; (d) FEM with time increment of $1.0 \times 10^{-3}, t=0.4$ Table 2 The relative errors between the meshless solution and the FEM solution

\begin{tabular}{ccccccccc}
\hline $\begin{array}{c}\text { Time increment } \\
\text { for FEM }\end{array}$ & $e_{\mathrm{s}}(0.1)$ & $e_{\mathrm{g}}(0.1)$ & $e_{\mathrm{s}}(0.2)$ & $e_{\mathrm{g}}(0.2)$ & $e_{\mathrm{s}}(0.3)$ & $e_{\mathrm{g}}(0.3)$ & $e_{\mathrm{s}}(0.4)$ & $e_{\mathrm{g}}(0.4)$ \\
\hline $1.0 \times 10^{-3}$ & 0.0189 & 0.0021 & 0.0084 & 0.0018 & 0.0104 & 0.0016 & 0.0119 & 0.0013 \\
& & & & & & & & 0.0091 \\
$1.0 \times 10^{-2}$ & 0.0576 & 0.0095 & 0.0393 & 0.0083 & 0.0336 & 0.0321 & 0.0107 \\
\hline
\end{tabular}

\section{Modeling of heat transfer during FSW}

\subsection{Problem description and numerical model}

FSW experiment for butt welding of Al 6061-T6 plates [7] is simulated. The workpieces in the experiment are two plates with a length of $254 \mathrm{~mm}$, a width of $102 \mathrm{~mm}$ and a thickness of $12.7 \mathrm{~mm}$. The tool material is H-13 tool steel. The tool pin's radius $r_{\mathrm{p}}$ and height $h_{\mathrm{p}}$ are $6 \mathrm{~mm}$ and $12 \mathrm{~mm}$, respectively. The tool shoulder's radius $r_{\mathrm{sh}}$ is $25 \mathrm{~mm}$.

As mentioned in Section 2.2, a half model is established to simulate the problem due to the symmetry. Fig. 8 shows the initial particle model, which includes $336804(254 \times 102 \times 13)$ uniform particles with a particle spacing of about $1 \mathrm{~mm}$. 


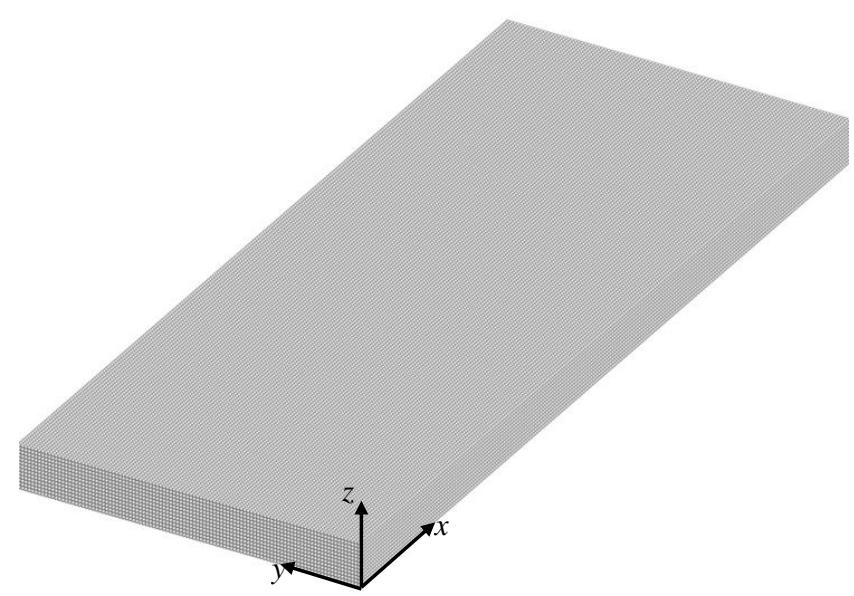

Fig. 8 The initial particle model

In the calculation, the smoothing length is 1.25 times of particle spacing, and the time step is $0.01 \mathrm{~s}$. For the heat generation model, both $t_{\mathrm{s}}$ and $t_{\mathrm{b}}$ are taken as $2 \mathrm{~mm}[3] . C_{\mathrm{f}}$ is estimated with

$$
C_{\mathrm{f}}=\lambda_{\mathrm{w}} /\left(\lambda_{\mathrm{w}}+\lambda_{\mathrm{T}}\right)
$$

and calibrated by comparing with numerical and experimental thermal cycles. The value of $C_{\mathrm{f}}$ used in this paper is 0.85 . For material properties, the density of Al6061-T6 is taken as $2700 \mathrm{~kg} / \mathrm{m}^{3}$, and the specific heat, the thermal conductivity and the yield stress of Al6061-T6 are considered to be temperature-dependent. The specific heat and thermal conductivity at specified temperatures are given by the following cubic polynomial

$$
a_{0}+a_{1} T+a_{2} T^{2}+a_{3} T^{3}
$$

where $a_{0}, a_{1}, a_{2}$ and $a_{3}$ are coefficients, whose values are given in Table 3 [49]. The yield stresses of Al6061-T6 at various temperatures are listed in Table 4 [6]. The yield stresses at intermediate temperatures are determined by linear interpolation.

Table 3 Coefficients for specific heat and conductivity [49]

\begin{tabular}{ccccc}
\hline Material property & $a_{0}$ & $a_{1}$ & $a_{2}$ & $a_{3}$ \\
\hline Specific heat $/ \mathrm{Jkg}^{-1} \mathrm{~K}^{-1}$ & $9.293 \times 10^{2}$ & $-6.27 \times 10^{-1}$ & $1.481 \times 10^{-3}$ & $-4.33 \times 10^{-8}$ \\
Conductivity $/ \mathrm{Wm}^{-1} \mathrm{~K}^{-1}$ & $2.522 \times 10^{1}$ & $3.978 \times 10^{-1}$ & $7.358 \times 10^{-6}$ & $-2.518 \times 10^{-7}$ \\
\hline
\end{tabular}

Table 4 Yield stress [6]

\begin{tabular}{ccccccccccc}
\hline Temperature/K & 311 & 339 & 366 & 394 & 422 & 450 & 477 & 533 & 589 & 644 \\
\hline Yield strength/MPa & 241 & 238 & 232 & 223 & 189 & 138 & 92 & 34 & 19 & 12 \\
\hline
\end{tabular}

\subsection{Results and discussion}

Fig. 9 shows the temperature distributions calculated by the present meshless particle method for different time in the welding process. Welding parameters are $637 \mathrm{r} / \mathrm{min}$ for the tool's rotational speed $(n)$ and $1.59 \mathrm{~mm} / \mathrm{s}$ for the welding speed $\left(v_{\mathrm{w}}\right)$. Fig. 9 (a) gives the result of $t=2 \mathrm{~s}$ when it is in the plunge process. The tool pin has penetrated into the workpiece for a distance, but the tool 
shoulder is still not in contact with the workpiece. Heat is only generated by the tool pin, so a cylindrical region with the highest level of temperature appears around the tool pin. Fig. 9(b) shows the result of $t=3 \mathrm{~s}$ when the tool shoulder has been in contact with the workpiece for about $0.5 \mathrm{~s}$. Heat generated by the tool shoulder becomes the main heat source, so the top surface of the workpiece within the tool shoulder is heated rapidly and shows the highest temperature. As the welding progresses, the temperature distribution in the welding region stabilizes gradually. From Figs. 9(e) and (f), it can be seen that there is little difference between the temperature distributions of $t=30 \mathrm{~s}$ and $t=40 \mathrm{~s}$ in the region around the tool pin. This indicates that the welding basically reaches a steady state.

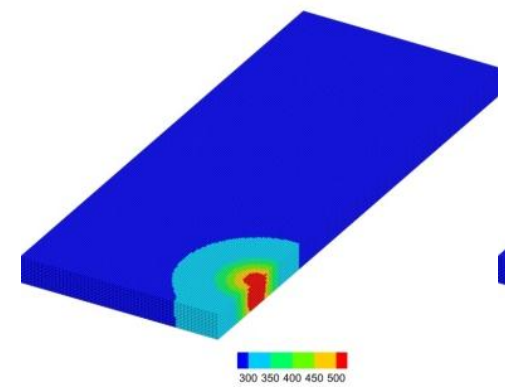

(a)

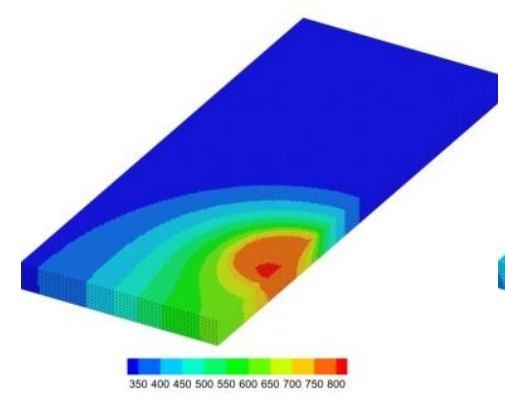

(d)

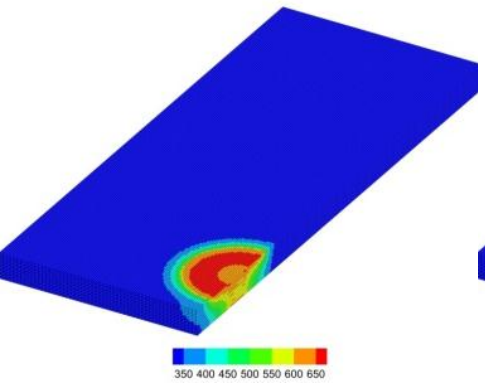

(b)

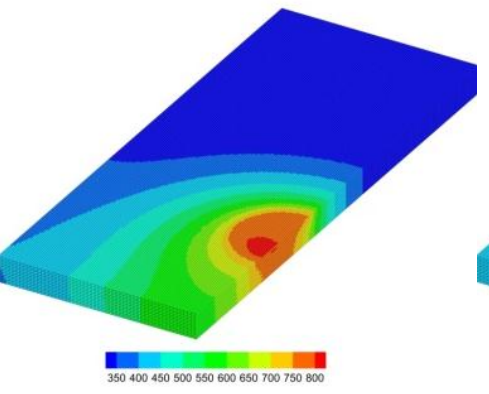

(e)

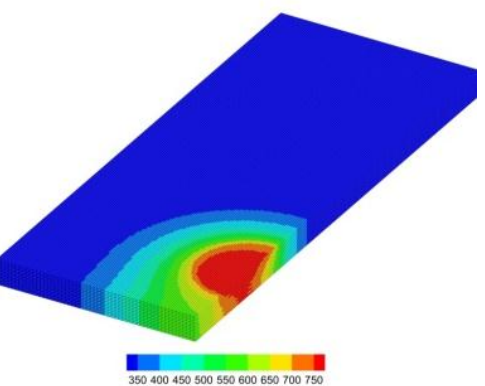

(c)

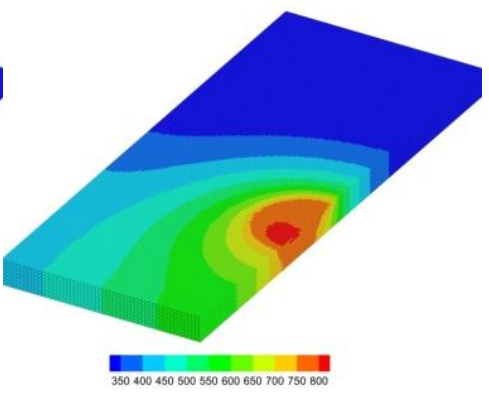

(f)

Fig. 9 Temperature distributions calculated by the present meshless method for different time: (a) $t=2 \mathrm{~s}$; (b) $t=3 \mathrm{~s}$; (c)

$$
t=10 \mathrm{~s} \text {; (d) } t=20 \mathrm{~s} \text {; (e) } t=30 \mathrm{~s} \text {; (f): } t=40 \mathrm{~s}
$$

For comparative purposes, a FEM model is established and solved with ABAQUS/Standard. The model has the same number of degrees of freedom as that of the previous meshless model. The heat generation model is implemented in the user subroutine DFLUX. Automatic incrementation is chosen for calculation. Initial time increment and maximum time increment are specified as 0.1 and 1 , respectively. Fig. 10 gives the temperature distributions calculated by FEM. It can be seen that the results are similar to those in Fig. 9. Whereas, the computational cost of the two methods is quite different. Under the same computer configurations (Intel (R) Xeon (R) CPU E5-1650, 32GB RAM), it takes about 200 hours to complete the FEM calculation, while only 21 hours are used to finish the meshless calculation. This is because the implicit FEM requires time-consuming iteration, and its remarkable advantage in time step is restrained due to the moving heat source and the intensive nonlinearity existing in the current problem. Fig. 11 shows the time increment for each increment during the FEM solving process. It can be observed that the time increment is restricted to about $0.04 \mathrm{~s}$ for most increments. 


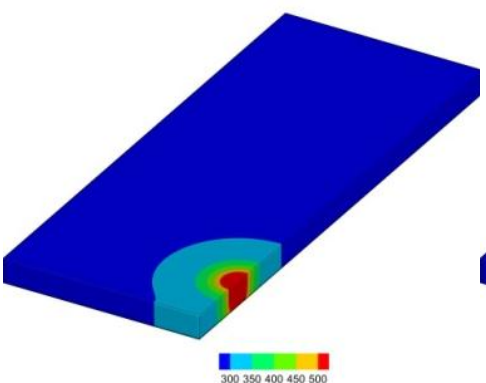

(a)

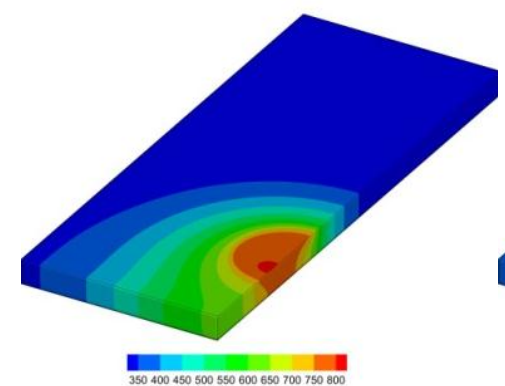

(d)

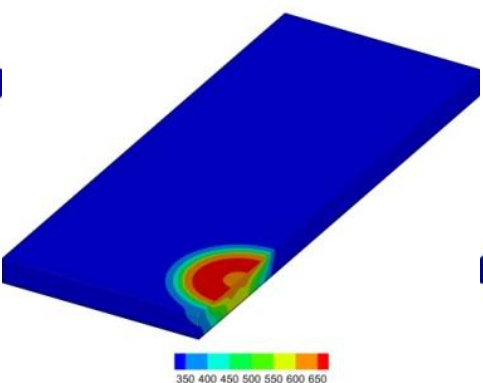

(b)

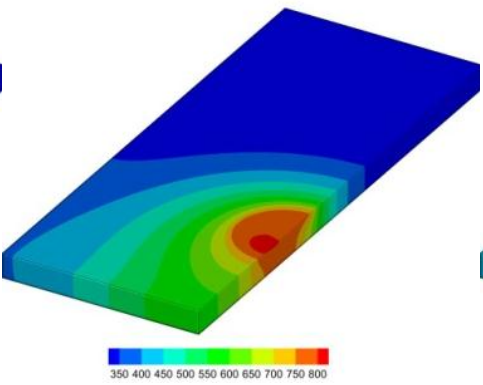

(e)

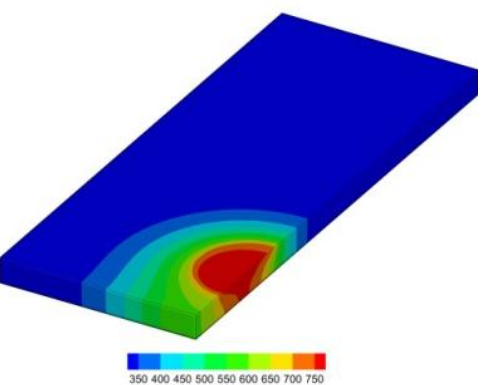

(c)

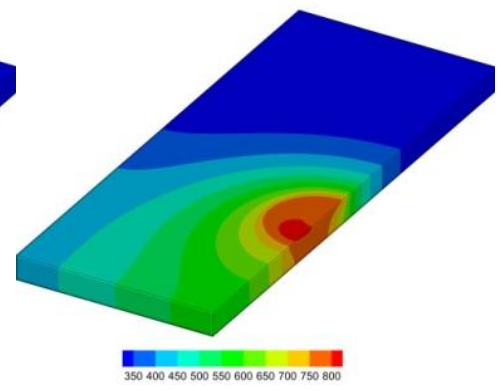

(f)

Fig. 10 Temperature distributions calculated by FEM for different time: (a) $t=2 \mathrm{~s}$; (b) $t=3 \mathrm{~s}$; (c) $t=10 \mathrm{~s}$; (d) $t=20 \mathrm{~s}$; (e) $t=30 \mathrm{~s} ;$ (f): $t=40 \mathrm{~s}$

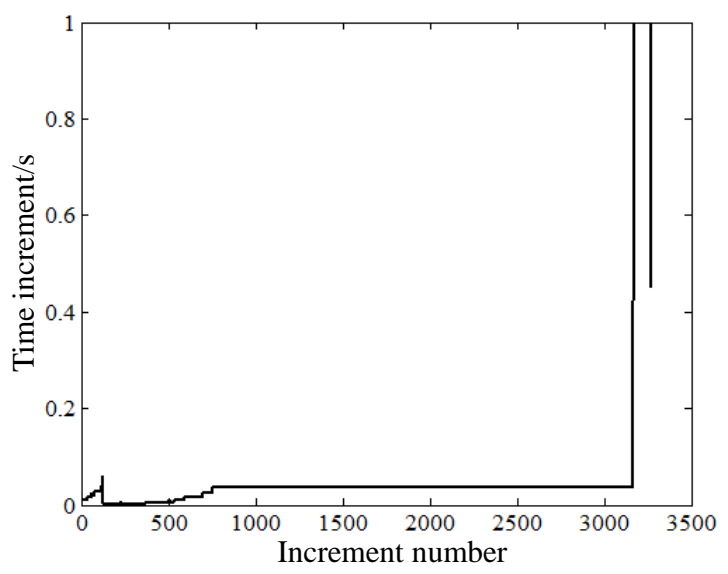

Fig. 11 Time increment for each increment

Figs. 12(a) to (d) shows a comparison between the calculated and experimental thermal cycles for four points. Points 1 and 2 are $2 \mathrm{~mm}$ below the top surface of the workpiece, and are $8 \mathrm{~mm}$ and $16 \mathrm{~mm}$ away from the weld line, respectively. Points 3 and 4 are $8 \mathrm{~mm}$ below the top surface, and are also $8 \mathrm{~mm}$ and $16 \mathrm{~mm}$ away from the weld line, respectively. It can be seen that the results calculated by the present method are in good agreement with the experimental ones. The peak temperatures of the four points computed by the present method are a little lower than the experimental ones. The maximum temperature difference is about $36 \mathrm{~K}$. 


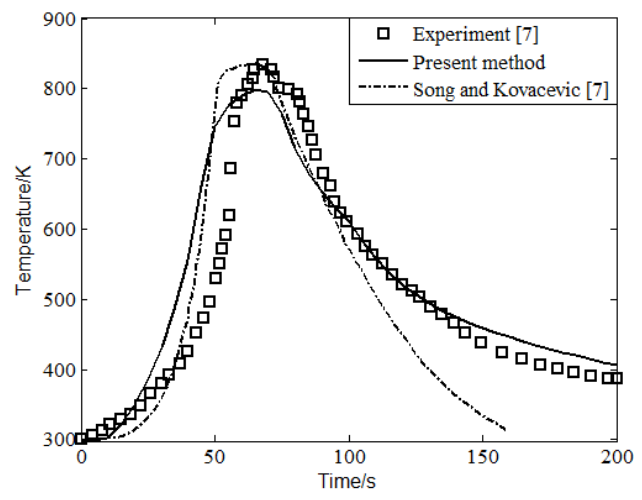

(a)

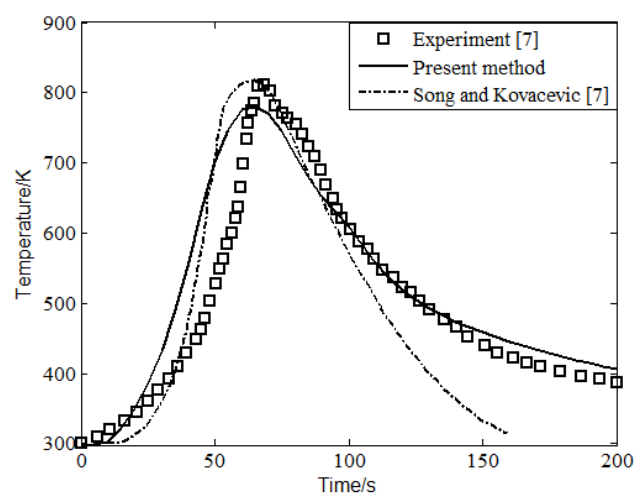

(c)

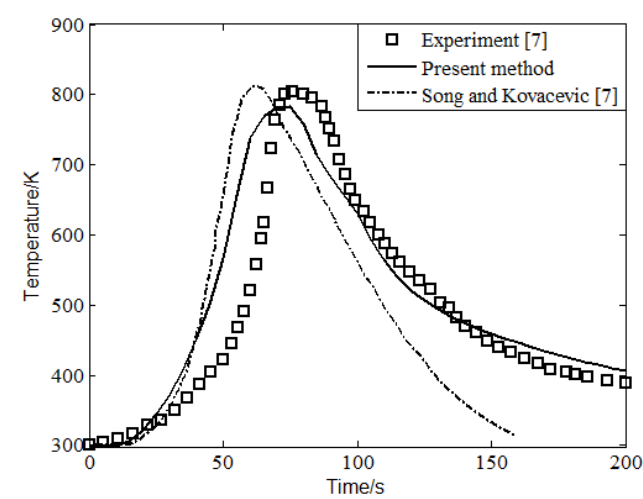

(b)

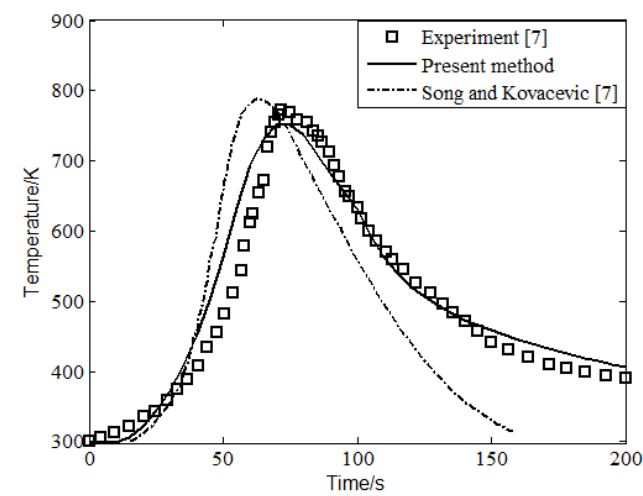

(d)

Fig. 12 Temperature cycles of four points: (a) Point 1; (b) Point 2; (c) Point 3; (d) Point 4

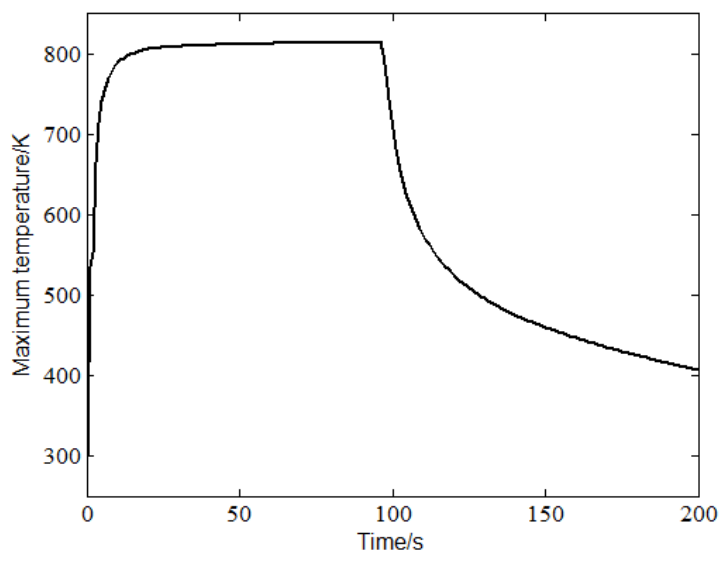

Fig. 13 The time history of maximum temperature in the workpiece

Fig. 13 shows the history of maximum temperature in the workpiece. The maximum temperature increases rapidly in the plunge stage, remains nearly unchanged in most of the welding stage, and decreases quickly after the tool is pulled out at the end of the welding stage. During the whole process, the maximum temperature in the workpiece is $815 \mathrm{~K}$, which doesn't exceed the melting point $855 \mathrm{~K}$. This agrees with the fact that FSW is a solid-state welding process.

Table 5 gives the maximum temperature of the workpiece $\left(T_{\max }\right)$ during the whole welding process, the heat generation rates of the tool pin's side, the tool pin's bottom surface and the 
shoulder $\left(P_{\mathrm{s}}, P_{\mathrm{b}}\right.$ and $\left.P_{\mathrm{sh}}\right)$, the welding heat input $(q)$ and the torque exerted on the tool $(M)$ for various welding speeds. $P_{\mathrm{s}}, P_{\mathrm{b}}, P_{\mathrm{sh}}, q$ and $M$ are calculated at $50 \mathrm{~s}$ when the welding basically reaches a steady state. It can be seen that $P_{\mathrm{s}}, P_{\mathrm{b}}$ and $P_{\mathrm{sh}}$ increase as $v_{\mathrm{w}}$ increases. Also, $M$ increases with the growth of $v_{\mathrm{w}}$. However, as $v_{\mathrm{w}}$ increases, the heat input $q$ decreases, so $T_{\max }$ becomes lower.

Table 5 Maximum temperature, heat generation rates, welding heat input and torque for different welding speeds

\begin{tabular}{cccccccc}
\hline$v_{\mathrm{w}} /(\mathrm{mm} / \mathrm{s})$ & $n /(\mathrm{r} / \mathrm{min})$ & $T_{\max } / \mathrm{K}$ & $P_{\mathrm{s}} / \mathrm{kW}$ & $P_{\mathrm{b}} / \mathrm{kW}$ & $P_{\mathrm{sh}} / \mathrm{kW}$ & $q /(\mathrm{kJ} / \mathrm{mm})$ & $M / \mathrm{Nm}$ \\
\hline 0.80 & 637 & 820 & 0.369 & 0.066 & 4.870 & 5.637 & 79.5 \\
1.59 & 637 & 815 & 0.419 & 0.075 & 5.447 & 3.176 & 89.1 \\
2.39 & 637 & 812 & 0.474 & 0.084 & 6.030 & 2.343 & 98.8 \\
\hline
\end{tabular}

Table 6 shows the calculated $T_{\max }, P_{\mathrm{s}}, P_{\mathrm{b}}, P_{\mathrm{sh}}, q$ and $M$ for different rotational speeds of the tool. It can be seen that the heat generation rates, $P_{\mathrm{s}}, P_{\mathrm{b}}$ and $P_{\mathrm{sh}}$, increase as the rotational speed $n$ increases. The heat input $q$ also increases as $n$ increases, since the welding speed keeps the same. Because of the increasing heat input $q$, the maximum temperature $T_{\max }$ becomes higher with the growth of $n$. However, the torque exerted on the tool decreases as $n$ gets larger.

Table 6 Maximum temperature, heat generation rates, welding heat input and torque for different rotational speeds

\begin{tabular}{cccccccc}
\hline$v_{\mathrm{w}} /(\mathrm{mm} / \mathrm{s})$ & $n /(\mathrm{r} / \mathrm{min})$ & $T_{\max } / \mathrm{K}$ & $P_{\mathrm{s}} / \mathrm{kW}$ & $P_{\mathrm{b}} / \mathrm{kW}$ & $P_{\mathrm{sh}} / \mathrm{kW}$ & $q /(\mathrm{kJ} / \mathrm{mm})$ & $M / \mathrm{Nm}$ \\
\hline 1.59 & 319 & 767 & 0.375 & 0.065 & 4.738 & 2.768 & 155.0 \\
1.59 & 637 & 815 & 0.419 & 0.075 & 5.447 & 3.176 & 89.1 \\
1.59 & 956 & 831 & 0.437 & 0.080 & 5.745 & 3.348 & 62.5 \\
\hline
\end{tabular}

Table 7 shows the calculated $T_{\max }, P_{\mathrm{s}}, P_{\mathrm{b}}, P_{\mathrm{sh}}, q$ and $M$ for different shoulder radii. It can be seen that $P_{\mathrm{sh}}$ increases as $r_{\mathrm{sh}}$ increases since the shoulder's area becomes larger. $P_{\mathrm{s}}$ and $P_{\mathrm{b}}$ decrease with the growth of $r_{\mathrm{sh}}$, because the area of the side and the bottom surface of the pin keeps the same while the frictional stresses on them decrease due to the higher temperature and the lower yield strength of the workpiece. The heat generated from the shoulder is dominant in the total heat generation. It takes up as much as $91.7 \%$ when $r_{\mathrm{sh}}$ is $25 \mathrm{~mm}$. With the growth of $r_{\mathrm{sh}}$, the maximum temperature $T_{\max }$ gets higher because of the increasing heat input $q$, and the torque exerted on the tool $M$ becomes larger.

Table 7 Maximum temperature, heat generation rates, welding heat input and torque for different shoulder radii

\begin{tabular}{|c|c|c|c|c|c|c|c|c|c|}
\hline \multirow{2}{*}{$r_{\mathrm{sh}} /(\mathrm{mm})$} & \multirow{2}{*}{$T_{\max } / \mathrm{K}$} & \multicolumn{2}{|c|}{$P_{\mathrm{s}}$} & \multicolumn{2}{|c|}{$P_{\mathrm{b}}$} & \multicolumn{2}{|c|}{$P_{\mathrm{sh}}$} & \multirow{2}{*}{$q /(\mathrm{kJ} / \mathrm{mm})$} & \multirow{2}{*}{$M / \mathrm{Nm}$} \\
\hline & & $\mathrm{kW}$ & $\%$ & $\mathrm{~kW}$ & $\%$ & $\mathrm{~kW}$ & $\%$ & & \\
\hline 15 & 735 & 0.999 & $28.2 \%$ & 0.172 & $4.9 \%$ & 2.378 & $67.0 \%$ & 1.897 & 53.2 \\
\hline 20 & 785 & 0.652 & $13.7 \%$ & 0.115 & $2.4 \%$ & 3.999 & $83.9 \%$ & 2.548 & 71.5 \\
\hline 25 & 815 & 0.419 & $7.1 \%$ & 0.075 & $1.3 \%$ & 5.447 & $91.7 \%$ & 3.176 & 89.1 \\
\hline
\end{tabular}

\section{Conclusions}

A meshless particle method is developed for modeling heat transfer during FSW process. The method is truly meshless and easy for implementation. It employs a particle approximation having first-order consistency to ensure good computational accuracy, uses a newly developed penalty method to impose boundary conditions accurately, and adopts a smoothing algorithm to enhance the numerical stability. The computational accuracy and parametric effect of the method are examined by two numerical examples in comparing with analytical solution and results obtained by FEM. Numerical results demonstrate that the method can achieve excellent accuracy with a 
stable time-step coefficient up to 0.5 and is not sensitive to the particle distribution. The method is successfully applied to the simulation of heat transfer during FSW of Al6061-T6 plates. The simulated temperature cycles are in reasonable agreement with experimental results. Simulation of the same problem using implicit FEM is also conducted. It is found that meshless and implicit FEM simulations yield similar results, but the former can save a lot of computational time. FSW process with various welding parameters is also numerically studied with the meshless method. Maximum temperature in the workpiece, heat generation rates of different parts of the tool, welding heat input and torque exerted on the tool are predicted.

\section{Acknowledgement}

The support from National Science Foundation of China (Grant No.: 11302077 and 21466012) and China Scholarship Council is gratefully acknowledged.

\section{References}

[1] B.T. Gibson, D.H. Lammlein, T.J. Prater, et al., Friction stir welding: process, automation, and control, Journal of Manufacturing Processes 16(1) (2014) 56-73.

[2] Y.J. Chao, X. Qi, W. Tang, Heat transfer in friction stir welding - experimental and numerical studies, Journal of manufacturing science and engineering 125(1) (2003) 138-145.

[3] M. Selvaraj, V. Murali, S.R. Koteswara Rao, Thermal model for friction stir welding of mild steel, Multidiscipline Modeling in Materials and Structures 9(1) (2013) 49-61.

[4] M. Abbasi, B. Bagheri, R. Keivani, Thermal analysis of friction stir welding process and investigation into affective parameters using simulation, Journal of Mechanical Science and Technology 29(2) (2015) 861-866.

[5] M. Song, R. Kovacevic, Numerical and experimental study of the heat transfer process in friction stir welding, Proceedings of the Institution of Mechanical Engineers, Part B: Journal of Engineering Manufacture 217(1) (2003) 73-85.

[6] M. Song, R. Kovacevic, Thermal modeling of friction stir welding in a moving coordinate system and its validation, International Journal of Machine Tools and Manufacture 43(6) (2003) 605-615.

[7] M. Song, R. Kovacevic, Heat transfer modelling for both workpiece and tool in the friction stir welding process: a coupled model, Proceedings of the Institution of Mechanical Engineers, Part B: Journal of Engineering Manufacture 218(1) (2004) 17-33.

[8] I. Alfaro, L. Fratini, E. Cueto, et al., Numerical simulation of friction stir welding by natural element methods, International Journal of Material Forming 1(1) (2008) 1079-1082.

[9] C.T. Wu, W. Hu, H.P. Wang, et al., A robust numerical procedure for the thermomechanical flow simulation of friction stir welding process using an adaptive element-free Galerkin method, Mathematical Problems in Engineering 2015 (2015) 1-16.

[10] A. Tartakovsky, G. Grant, X. Sun, et al., Modeling of friction stir welding (FSW) process with smooth particle hydrodynamics (SPH), In: SAE 2006 World Congress, Detroit, USA, 2006.

[11] S. Bhojwani, Smoothed particle hydrodynamics modeling of the friction stir welding process, Mater thesis, The University of Texas at El Paso, El Paso, Texas, 2007.

[12] W. Pan, D. Li, A.M. Tartakovsky, et al., A new smoothed particle hydrodynamics non-Newtonian model for friction stir welding: Process modeling and simulation of microstructure evolution in a magnesium alloy, International Journal of Plasticity 48 (2013) 189-204.

[13] K. Fraser, L. St-Georges, L.I. Kiss, Prediction of defects in a friction stir welded joint using the smoothed particle hydrodynamics method, in: 7th Asia Pacific IIW International Congress, Singapore, 2013.

[14] R.A. Gingold, J.J. Monaghan, Smoothed particle hydrodynamics: theory and application to non-spherical 
stars, Monthly notices of the royal astronomical society 181(3) (1977) 375-389.

[15] T. Belytschko, Y.Y. Lu, L. Gu, element-free Galerkin methods, International journal for numerical methods in engineering 37(2) (1994) 229-256.

[16] W.K. Liu, S. Jun, Y.F. Zhang, Reproducing kernel particle methods, International journal for numerical methods in fluids 20 (1995) 1081-1106.

[17] S.N. Atluri, T. Zhu, A new meshless local Petrov-Galerkin (MLPG) approach in computational mechanics, Computational mechanics 22(2) (1998) 117-127.

[18] G.R. Liu, Y.T. Gu, A point interpolation method for two-dimensional solids, International Journal for Numerical Methods in Engineering 50(4) (2001) 937-951.

[19] Y.L. Wu, G.R. Liu, Y.T. Gu, Application of meshless local Petrov-Galerkin (MLPG) approach to simulation of incompressible flow, Numerical Heat Transfer, Part B: Fundamentals 48(5) (2005) 459-475.

[20] Y.H. Xiao, D.A. Hu, X. Han, et al., Simulation of normal perforation of aluminum plates using axisymmetric smoothed particle hydrodynamics with contact algorithm, International Journal of Computational Methods 10(3) (2013) 1350039.

[21] G. Yang, Y.K. Fu, D.A. Hu, et al., Feasibility analysis of SPH method in the simulation of condensed explosives detonation with ignition and growth model, Computers \& Fluids 88 (2013) 51-59.

[22] A. Singh, I.V. Singh, R. Prakash, Meshless element free Galerkin method for unsteady nonlinear heat transfer problems, International Journal of Heat and Mass Transfer 50(5) (2007) 1212-1219.

[23] A. Singh, I.V. Singh, R. Prakash, Meshless analysis of unsteady-state heat transfer in semi-infinite solid with temperature-dependent thermal conductivity, International communications in heat and mass transfer 33(2) (2006) 231-239.

[24] A. Singh, I.V. Singh, R. Prakash, Numerical solution of temperature-dependent thermal conductivity problems using a meshless method, Numerical Heat Transfer, Part A: Applications 50(2) (2006) 125-145.

[25] I.V. Singh, A numerical study of weight functions, scaling, and penalty parameters for heat transfer applications, Numerical Heat Transfer, Part A: Applications 47(10) (2005) 1025-1053.

[26] I.V. Singh, A numerical solution of composite heat transfer problems using meshless method, International Journal of Heat and Mass Transfer 47(10) (2004) 2123-2138.

[27] I.V. Singh, Heat transfer analysis of composite slabs using meshless element free Galerkin method, Computational Mechanics 38(6) (2006) 521-532.

[28] I. V. Singh, M. Tanaka, Thermal solution of cylindrical composite systems using meshless method, Heat and mass transfer 42(8) (2006) 689-707.

[29] I.V. Singh, M. Tanaka, M. Endo, Meshless method for nonlinear heat conduction analysis of nano-composites, Heat and mass transfer 43(10) (2007) 1097-1106.

[30] I.V. Singh, M. Tanaka, M. Endo, Thermal analysis of CNT-based nano-composites by element free Galerkin method, Computational Mechanics 39(6) (2007) 719-728.

[31] I.V. Singh, M. Tanaka, M. Endo, Effect of interface on the thermal conductivity of carbon nanotube composites, International journal of thermal sciences 46(9) (2007) 842-847.

[32] I.V. Singh, M. Tanaka, M. Endo, Nonlinear thermal analysis of carbon nanotube composites by element free Galerkin method, Numerical Heat Transfer, Part A: Applications 51(11) (2007) 1087-1102.

[33] X.H. Wu, W.Q. Tao, Meshless method based on the local weak-forms for steady-state heat conduction problems, International Journal of Heat and Mass Transfer 51(11) (2008) 3103-3112.

[34] H. Thakur, K.M. Singh, P.K. Sahoo, MLPG analysis of nonlinear heat conduction in irregular domains, Computer Modeling in Engineering and Sciences (CMES) 68(2) (2010) 117.

[35] H. Thakur, K.M. Singh, P.K. Sahoo, Phase change problems using the MLPG method, Numerical Heat Transfer, Part A: Applications 59(6) (2011) 438-458. 
[36] J. Tian, S.S. Rao, Meshless local Petrov-Galerkin method for three-dimensional heat transfer analysis, Journal of Heat Transfer 134(11) (2012) 112701.

[37] J.K. Chen, J.E. Beraun, C.J. Jih, An improvement for tensile instability in smoothed particle hydrodynamics, Computational Mechanics 23(4) (1999) 279-287.

[38] M.B. Liu, W.P. Xie, G.R. Liu, Modeling incompressible flows using a finite particle method, Applied mathematical modelling 29(12) (2005) 1252-1270.

[39] G.M. Zhang, R.C. Batra, Modified smoothed particle hydrodynamics method and its application to transient problems, Computational mechanics 34(2) (2004) 137-146.

[40] G.M. Zhang, R.C. Batra, SSPH basis functions for meshless methods, and comparison of solutions with strong and weak formulations, Computational Mechanics 41(4) (2008) 527-545.

[41] J.J. Monaghan, H.E. Huppert, M.G. Worster, Solidification using smoothed particle hydrodynamics, Journal of Computational Physics 206(2) (2005) 684-705.

[42] J.H. Jeong, M.S. Jhon, J.S. Halow, et al., Smoothed particle hydrodynamics: Applications to heat conduction, Computer Physics Communications 153(1) (2003) 71-84.

[43] Y.H. Xiao, H.F. Zhang, X.C. Ping, et al., 3D simulation of temperature field during welding of stainless steels with meshless symmetric particle method, China Mechanical Engineering 26(24) (2015) 3336-3340.

[44] H.B. Schmidt, J. H. Hattel, Thermal modelling of friction stir welding, Scripta Materialia 58(5) (2008) 332-337.

[45] J.J. Monaghan, On the problem of penetration in particle methods, Journal of Computational physics 82(1) (1989) $1-15$.

[46] G.R. Liu, M.B. Liu, Smoothed particle hydrodynamics: a meshfree particle method, World Scientific, Singapore, 2003, pp. 208-209.

[47] J. Fourier, The analytical theory of heat, Translated with notes by Alexander Freeman, Cambridge University Press, London, 1878, pp. 326-327.

[48] Abaqus 6.10 Documentation, Dassault Systèmes, 2010.

[49] R. Nandan, G.G. Roy, T. Debroy, Numerical simulation of three-dimensional heat transfer and plastic flow during friction stir welding, Metallurgical and Materials Transactions A 37(4) (2006) 1247-1259. 